\title{
Complex Boundary Value Techniques \\ Applied to Two Queue Networks \\ With Coupled Processing
}

\section{Keith Oliver, B.Eng.}

\author{
A thesis \\ Submitted to the Faculty of Graduate Studies and Research \\ Carleton University \\ In partial fulfillment of the requirements \\ For the degree of Master of Science in the \\ Ottawa-Carleton Institute of Mathematics and Statistics
}

(C) Keith Oliver

September 2007 


$\begin{array}{ll}\begin{array}{l}\text { Library and } \\ \text { Archives Canada }\end{array} & \begin{array}{l}\text { Bibliothèque et } \\ \text { Archives Canada }\end{array} \\ \begin{array}{l}\text { Published Heritage } \\ \text { Branch }\end{array} & \begin{array}{l}\text { Direction du } \\ \text { Patrimoine de l'édition }\end{array} \\ \begin{array}{l}\text { 395 Wellington Street } \\ \text { Ottawa ON K1A ON4 }\end{array} & \begin{array}{l}\text { 395, rue Wellington } \\ \text { Ottawa ON K1A ON4 } \\ \text { Canada }\end{array}\end{array}$

Your file Votre référence ISBN: 978-0-494-33710-3 Our file Notre référence ISBN: $978-0-494-33710-3$

NOTICE:

The author has granted a nonexclusive license allowing Library and Archives Canada to reproduce, publish, archive, preserve, conserve, communicate to the public by telecommunication or on the Internet, loan, distribute and sell theses worldwide, for commercial or noncommercial purposes, in microform, paper, electronic and/or any other formats.

The author retains copyright ownership and moral rights in this thesis. Neither the thesis nor substantial extracts from it may be printed or otherwise reproduced without the author's permission.
AVIS:

L'auteur a accordé une licence non exclusive permettant à la Bibliothèque et Archives Canada de reproduire, publier, archiver, sauvegarder, conserver, transmettre au public par télécommunication ou par l'Internet, prêter, distribuer et vendre des thèses partout dans le monde, à des fins commerciales ou autres, sur support microforme, papier, électronique et/ou autres formats.

L'auteur conserve la propriété du droit d'auteur et des droits moraux qui protège cette thèse. $\mathrm{Ni}$ la thèse ni des extraits substantiels de celle-ci ne doivent être imprimés ou autrement reproduits sans son autorisation.
In compliance with the Canadian

Privacy Act some supporting forms may have been removed from this thesis.

While these forms may be included in the document page count, their removal does not represent any loss of content from the thesis.
Conformément à la loi canadienne sur la protection de la vie privée, quelques formulaires secondaires ont été enlevés de cette thèse.

Bien que ces formulaires aient inclus dans la pagination, il n'y aura aucun contenu manquant.

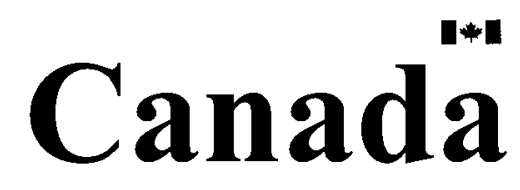




\section{Abstract}

Two examples are presented in this thesis that demonstrate how for a network with two queues, the queue length distribution can be characterized and performance measures calculated using complex analysis. The two models analyzed are

1. A symmetric "join the shortest queue" model with two, parallel, coupled processors, and

2. A two-node Jackson network with coupled processing

For each model, a functional equation is found that provides a relationship through which the characteristic function can be derived as the solution to a complex boundary value problem. A concise form for the average queue lengths is also presented. 


\section{Acknowledgements}

I would like to thank my supervisor Prof. Yiqiang Zhao for his assistance on this thesis. I appreciate his professional manner and the balance he achieved between fostering the ability to research in an independent manner with the need to provide timely direction to a novice. I am pleased that I was given such a challenging topic for a Master's thesis. For taking me on as a student when he was already carrying a heavy workload, including his responsibilities as director of the School, I am grateful.

Unfortunately, my studies at Carleton have been marred by the passing of two individuals. The first is Prof. Amit Bose who played a large part in convincing me to enroll in the Math \& Stats program at Carleton. He taught me stochastics and kindled my desire to research this field. He always took a keen interest in my affairs.

Early in my studies for this degree, my dear wife Chantal tragically died in a traffic accident. Ironically, this was within a few weeks of completing her own Master's thesis defense. She was always clear about her desire to see me continue in my studies and always proclaimed confidence in my abilities even when I had doubts. She will be loved forever.

Thank you to my friends and family for motivating me to work hard, distracting me when needed, and trying to understand why I found "line-ups" so fascinating. 


\section{Dedication}

To my late wife, Chantal 


\section{Contents}

1 Introduction 1

2 Coupled - JSQ 4

2.1 Model Description ................. . . 4

2.1.1 The Balance Equations . . . . . . . . . . . . . 6

2.1 .2 The Functional Equation . . . . . . . . . . . . 8

2.2 Preliminary Results . . . . . . . . . . . . . . . . . 11

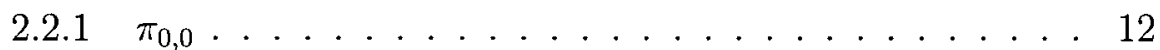

2.2.2 A Relationship between $\mathbb{E} Y_{1}$ and $\mathbb{E} Y_{2} \ldots \ldots \ldots$

2.2.3 Individual Forms for $\mathbb{E} Y_{1}$ and $\mathbb{E} Y_{2} \ldots \ldots \ldots$

2.3 A Boundary Value Problem Solution for $P(0, y) \ldots \ldots$

2.3.1 Analysis of the Kernel . . . . . . . . . . . . 19

2.3.2 Analytic Continuation ... . . . . . . . . . 24

2.3.3 The Boundary Value Problem ... . . . . . . . 26

2.3.4 Determination of the Conformal Mapping and its Inverse 27

2.4 A Boundary Value Problem Solution for $P(x, 0) \ldots 30$

2.4.1 Analysis of the Kernel . . . . . . . . . . . . 30

2.4 .2 Analytic Continuation ............. 34

2.4.3 The Boundary Value Problem . . . . . . . . . . 35

2.5 Conclusion ...................... 36

3 Coupled Jackson $\quad 38$

3.1 Model Description . . . . . . . . . . . . . . . . 38

3.1.1 The Balance Equations . . . . . . . . . . . . 40

3.1 .2 The Functional Equation . . . . . . . . . . . . . 42

3.2 Some Preliminary Results . . . . . . . . . . . . . . . . . 46

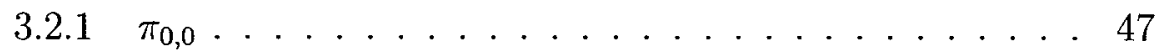

3.2.2 A Relationship between $\mathbb{E} X_{1}$ and $\mathbb{E} X_{2} \ldots \ldots . \ldots 51$ 
3.2.3 Individual Forms for $\mathbb{E} X_{1}$ and $\mathbb{E} X_{2} \ldots \ldots \ldots 51$

3.2.4 When $p^{*}=0$ or $p^{*}=1 \ldots \ldots \ldots \ldots$

3.3 The Boundary Value Problem . . . . . . . . . . . . 54

3.3 .1 Analysis of the Kernel . . . . . . . . . . . . 54

3.3.2 Analytic Continuation ............... 61

3.3.3 The Boundary Value Problem . . . . . . . . . . . . 62

3.4 Conclusion . . . . . . . . . . . . . . 63 


\section{List of Figures}

2.1 Coupled - JSQ: Model Description . . . . . . . . . . . 5

2.2 Coupled - JSQ: State Transition Rates . . . . . . . . . . 7

2.3 Coupled - JSQ: Discriminant as a Function of Real $x \ldots \ldots 21$

2.4 Coupled - JSQ: The Contour $L \ldots \ldots \ldots . \ldots . . \ldots 23$

2.5 Coupled - JSQ: The Conformal Mapping to the Unit Circle . . 28

2.6 Coupled - JSQ: The Discriminant as a Function of Real y . . . 30

2.7 Coupled - JSQ: The Contour $R \ldots \ldots . \ldots . . \ldots 32$

3.1 Coupled Jackson: Model Description . . . . . . . . . . . . . 39

3.2 Coupled Jackson: State Transition Rates . . . . . . . . . . . . 41

3.3 Coupled Jackson: Discriminant as a Function of Real $x$. . . 56

3.4 Coupled Jackson: Three options for $D(1)=0 \ldots \ldots$. . . . 58

3.5 Coupled Jackson: The Contour $L \ldots$. . . . . . . . . . 60 


\section{Chapter 1}

\section{Introduction}

Markov processes on a one-dimensional state space are well understood. Textbooks have been written and university courses are taught on the behavior of such processes. On the other hand, while much may be known about two-dimensional Markov processes, methods of analysis yielding useful performance metrics are somewhat limited. Developing such useful techniques is a current topic of research. The purpose of this thesis is to provide two examples of how one such technique can be used.

The analytic technique to be demonstrated is based on complex analysis. Specifically, it uses the method of solving Riemann-Hilbert boundary value problems to characterize a two queue network.

If two queues in a network do not operate independently, the analysis of the distribution of their queue lengths becomes quite involved as standard one-dimensional queuing theory does not suffice. Complex boundary value problems can be formulated, however, the solutions to which fully characterize the queuing behavior.

At the core of such analysis is the characteristic function of the process. For a two-dimensional, stationary, Markov process, $X(t)=\left(X_{1}(t), X_{2}(t)\right)$, representing the length of two queues, with state space $\{(m, n): m, n=0,1,2,3, \ldots\}$, the characteristic function is defined for $x, y \in \mathbb{C}$ with $|x|,|y| \leq 1$ as

$$
\begin{aligned}
P(x, y) & =\mathbb{E}\left[x^{X_{1}} y^{X_{2}}\right] \\
& =\sum_{m=0}^{\infty} \sum_{n=0}^{\infty} \pi_{m, n} x^{m} y^{n}
\end{aligned}
$$


where,

$$
\pi_{m, n}=\mathbb{P}\left[X_{1}(t)=m, X_{2}(t)=n\right]
$$

is the stationary queue length distribution. For $|x|,|y|<1, P(x, y)$ is analytic. For fixed $x$ with $|x| \leq 1, P(x, y)$ is continuous in $y$ where $|y| \leq 1$. Likewise, for fixed $y$ with $|y| \leq 1, P(x, y)$ is continuous in $x$ where $|x| \leq 1$. Only for the most trivial models would it be possible to determine $P(x, y)$ from simply a probabilistic consideration of the problem. In the models considered in this thesis a functional equation is derived that relates $P(x, y)$ with $P(x, 0), P(0, y)$ and $P(0,0)$ as follows

$$
h_{1}(x, y) P(x, y)=h_{2}(x, y) P(x, 0)+h_{3}(x, y) P(0, y)+h_{4}(x, y) P(0,0)
$$

where

$$
P(x, 0)=\sum_{m=0}^{\infty} \pi_{m, 0} x^{m}, \quad P(0, y)=\sum_{n=0}^{\infty} \pi_{0, n} y^{n}, \quad P(0,0)=\pi_{0,0}
$$

If $h_{i}(x, y)$ for $i=1,2,3,4$ are known, to derive $P(x, y)$, it suffices to find $P(x, 0), P(0, y)$ and $P(0,0)$.

The functions $P(x, 0)$ and $P(0, y)$ are found as the solutions to complex boundary value problems. The basic principle used to formulate these problems is that if $h_{1}(x, y)=0$ when $P(x, y)$ is analytic, then the right hand side of (1.3) must also be zero. That is, for zero-pairs $(x, y)$ of $h_{1}(x, y)$ for which $P(x, y)$ is finite, it must be true that

$$
h_{2}(x, y) P(x, 0)+h_{3}(x, y) P(0, y)+h_{4}(x, y) P(0,0)=0
$$

This means that there is a region on which a relationship between $P(x, 0)$ and $P(0, y)$ can be expressed. A boundary condition is subsequently formed. The behavior of $P(x, 0)$ and $P(0, y)$ on the boundary is used to derive the function values within the boundary.

A commonly sought (although, certainly not the only) performance measure for queuing networks is the average queue length. It is easily seen that

$$
\frac{\mathrm{d}}{\mathrm{d} x} P(x, 1)=\sum_{m=0}^{\infty} \sum_{n=0}^{\infty} m x^{m-1} \pi_{m, n}
$$

so,

$$
\mathbb{E} X_{1}=\left[\frac{\mathrm{d}}{\mathrm{d} x} P(x, 1)\right]_{x=1}
$$


and, likewise,

$$
\mathbb{E} X_{2}=\left[\frac{\mathrm{d}}{\mathrm{d} y} P(1, y)\right]_{y=1}
$$

This means that knowing that functional equation and solving the boundary value problems to reveal $P(x, 0)$ and $P(0, y)$ is all that is required to compute the expected queue lengths.

The two queuing networks considered in this thesis are:

1. A "join the shortest queue" model with two parallel, symmetric queues with coupled processing, and

2. A two node Jackson network with coupled processing

It is the belief of the author that the first model has never been considered in literature before.

Both models use coupled processing. This means that the service rate of one queue will increase if the other queue becomes idle. Qualitatively, coupling can be viewed as a form of resource sharing. The resource allocated to servicing the queues is limited. When both queues are active, the resource must be shared between the two; but when one of the queues is inactive, all, or an increased portion of the resource may be allocated to the active queue.

The complex analysis technique of solving boundary problems was first applied to queueing system analysis by Fayolle and Iasnogorodski in their respective Ph.D. theses [3] and [8]. A subsequent joint paper by both authors [4] uses the boundary value technique to analyze two parallel queues with coupled processing. Cohen \& Boxma have written a thorough, albeit mathematically intimidating, text on the subject [2].

The analytic technique used in this thesis is based heavily on Chapter 9 of van Leeuwaarden's Ph.D. thesis [11]. The analysis of two tandem coupled queues is considered therein. The paper [13] by Resing describes this same model. Earlier work by Blanc [1] presents the analysis of uncoupled tandem queues. 


\section{Chapter 2}

\section{Join the Shortest Queue with Coupled Processors}

In this chapter a two queue network is analyzed. The characteristic function of the long-run queue length probability distribution is derived as the solution to a complex boundary value problem. Also, some key performance metrics are found. Significantly, the average queue length is presented.

\subsection{Model Description}

The two queue network under consideration is a variation of the standard "join the shortest queue" (JSQ) model. In the standard model, when customers arrive, they join the shorter of two parallel queues. If both queues have the same number of customers waiting then a new arrival joins either queue with equal probability. The single source of arrivals follows a Poisson process with rate $\lambda$. Both queues serve customers with the same exponentially distributed service time with rate $\nu$. After service is complete at either queue, customers leave the system.

The variation to this classical JSQ model introduced in this thesis is to couple the servicing of both queues. When both queues are serving customers, the service rate for both remains $\nu$. When one of the queues is empty, though, the rate of the other increases to $\nu^{*}$.

Joining the shortest queue is a model that has great "real-world" application. It can represent shoppers in a grocery store choosing a cashier with the 


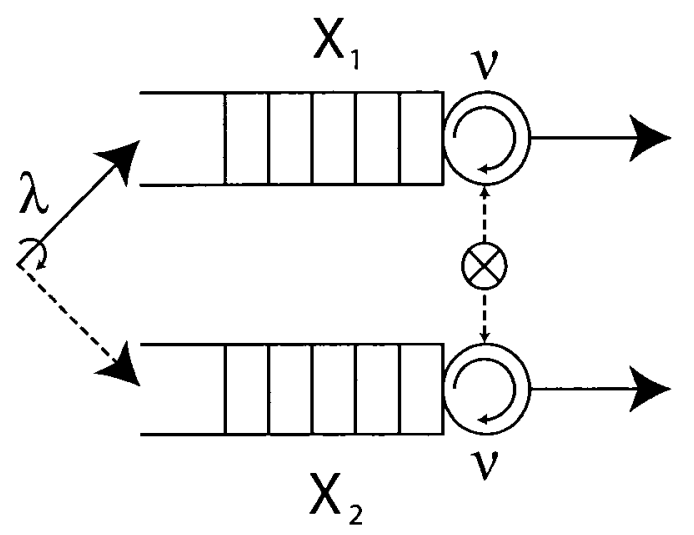

Figure 2.1: Coupled - JSQ: Model Description

shortest line, commuters choosing to enter a subway by the least crowded door, or, travelers at a border crossing selecting the customs official with the shortest queue. In computer science joining the shortest queue is an effective processor sharing algorithm - instructions are sent to the processor that is the least occupied.

It is also easy to think of analogies for coupled servicing within a JSQ network. Consider the grocery store where customers join the shortest line and the cashier that happens to have no customers waiting will assist another cashier by say, bagging groceries. Even though a computer may have two processors, both processors may have to share access to memory. Instructions that access memory will clearly be executed faster if only one processor is active.

It should come as no surprise, therefore, that the JSQ network has be studied extensively in literature. Two of the earliest papers are by Haight [7] and by Kingman [9]. The work of Flatto and McKean [5] uses complex analysis to find the characteristic function of the two-dimensional queue length distribution. Additionally they derive the equilibrium probabilities and mean queue lengths.

The boundary value problem technique used in this thesis has been applied to the uncoupled JSQ network by Cohen \& Boxma [2] in Section III.

It seems that this thesis is the first "work" to address the affect of coupled servicing on joining the shortest queue. 


\subsubsection{The Balance Equations}

Let $X_{1}(t)$ and $X_{2}(t)$ be the respective lengths of the two queues at time $t$. Analysis of this model is greatly simplified if, instead of analyzing $X_{1}(t)$ and $X_{2}(t)$ directly, $Y_{1}(t)$ and $Y_{2}(t)$ are considered, where

$$
Y_{1}(t)=\min \left(X_{1}(t), X_{2}(t)\right)
$$

and

$$
Y_{2}(t)=\left|X_{1}(t)-X_{2}(t)\right|
$$

The random process $Y(t)=\left(Y_{1}(t), Y_{2}(t)\right)$ is a Markov process that is irreducible and aperiodic. Let $\pi_{m, n}=\lim _{t \rightarrow \infty} \mathbb{P}\left[Y_{1}(t)=m, Y_{2}(t)=n\right]$ with $m, n=0,1,2,3, \ldots$. If $Y(t)$ is ergodic (which it will be if $\nu$ is sufficiently large for $\lambda$. See Lemma 2.2.1.) then $\pi\left(\pi=\left\{\pi_{m, n}: m, n=0,1,2,3, \ldots\right\}\right)$ exists and is the unique stationary distribution. Figure 2.2 illustrates the long-run state transition rates for $Y(t)$. Notice how the coupled service rate $\nu^{*}$ applies when $Y_{1}=0$; i.e. when at least one of the queues is empty.

For a stationary Markov process, a series of balance equations can be derived that fully characterize the process. These equations are found by recognizing that the rate at which the process enters a state must equal the rate at which it leaves that state. The stationary distribution $\pi$ is the unique solution to this system of equations.

In Figure 2.2, the shaded regions group states that have the same pattern of ingress and egress rates. Each shaded region has a corresponding balance equation, as follows:

For $m=1,2,3, \ldots$ and $n=2,3,4, \ldots$

$$
\begin{aligned}
\lambda \pi_{0,0} & =\nu^{*} \pi_{0,1} \\
\left(\lambda+\nu^{*}\right) \pi_{0,1} & =\lambda \pi_{0,0}+2 \nu \pi_{1,0}+\nu^{*} \pi_{0,2} \\
\left(\lambda+\nu^{*}\right) \pi_{0, n} & =\nu \pi_{1, n-1}+\nu^{*} \pi_{0, n+1} \\
(\lambda+2 \nu) \pi_{m, 0} & =\lambda \pi_{m-1,1}+\nu \pi_{m, 1} \\
(\lambda+2 \nu) \pi_{m, 1} & =\lambda \pi_{m, 0}+\lambda \pi_{m-1,2}+\nu \pi_{m, 2}+2 \nu \pi_{m+1,0} \\
(\lambda+2 \nu) \pi_{m, n} & =\lambda \pi_{m-1, n+1}+\nu \pi_{m, n+1}+\nu \pi_{m+1, n-1}
\end{aligned}
$$




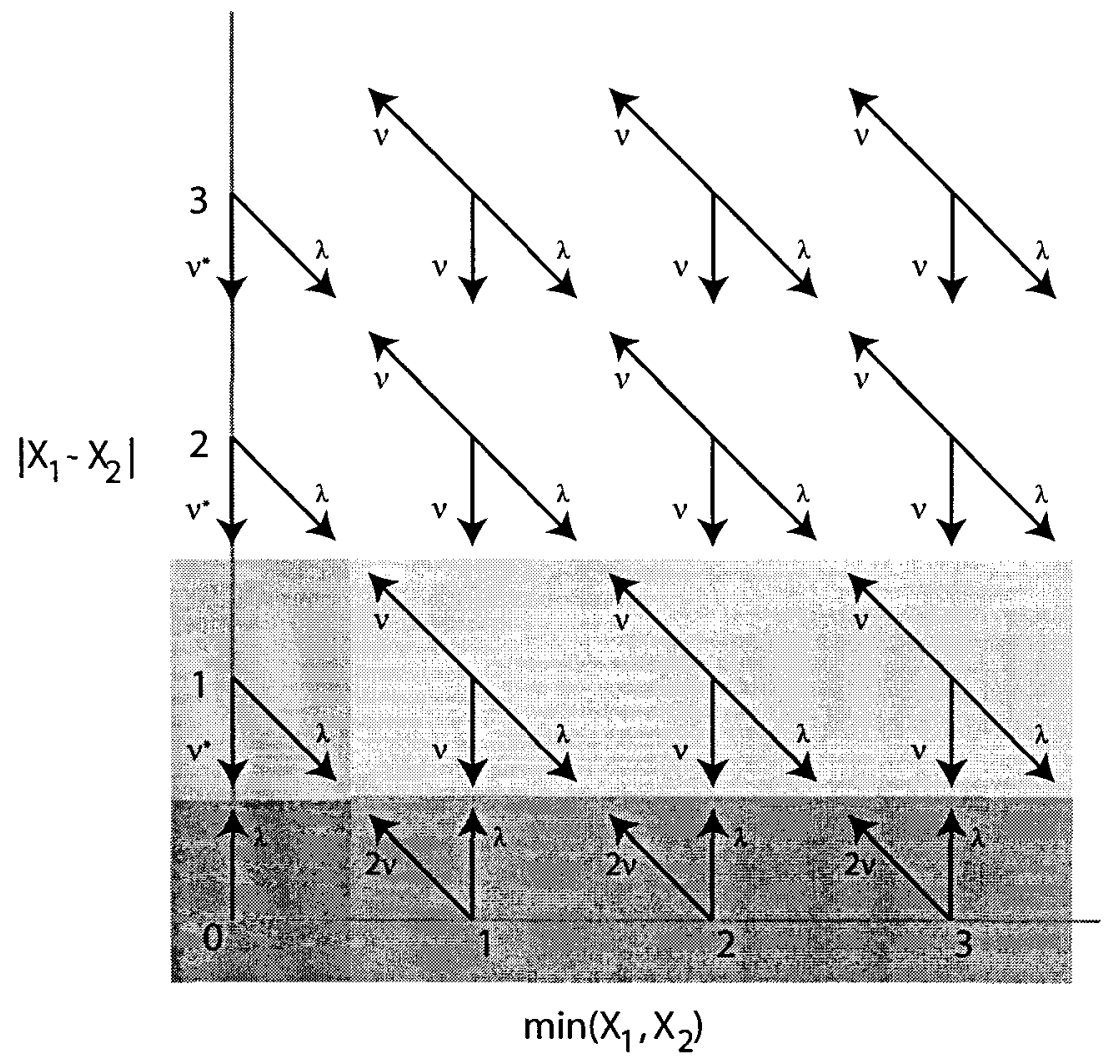

Figure 2.2: Coupled - JSQ: State Transition Rates 


\subsubsection{The Functional Equation}

In a very tedious, and error prone manner the balance equations can be collated to form the functional equation (1.3) for this network.

First, if (2.8) is multiplied by $x^{m+1} y^{n+1}$ and then summed for all valid states (i.e. $m=1,2,3, \ldots, n=2,3,4, \ldots$ ) it becomes

$$
\begin{aligned}
& (\lambda+2 \nu) \sum_{m=1}^{\infty} \sum_{n=2}^{\infty} x^{m+1} y^{n+1} \pi_{m, n}= \\
& \quad \lambda \sum_{m=1}^{\infty} \sum_{n=2}^{\infty} x^{m+1} y^{n+1} \pi_{m-1, n+1}+\nu \sum_{m=1}^{\infty} \sum_{n=2}^{\infty} x^{m+1} y^{n+1} \pi_{m, n+1} \\
& \quad+\nu \sum_{m=1}^{\infty} \sum_{n=2}^{\infty} x^{m+1} y^{n+1} \pi_{m+1, n-1}
\end{aligned}
$$

or,

$$
\begin{aligned}
& (\lambda+2 \nu) x y \sum_{m=1}^{\infty} \sum_{n=2}^{\infty} x^{m} y^{n} \pi_{m, n}= \\
& \lambda x^{2} \sum_{m=0}^{\infty} \sum_{n=3}^{\infty} x^{m} y^{n} \pi_{m, n}+\nu x \sum_{m=1}^{\infty} \sum_{n=3}^{\infty} x^{m} y^{n} \pi_{m, n} \\
& \quad+\nu y^{2} \sum_{m=2}^{\infty} \sum_{n=1}^{\infty} x^{m} y^{n} \pi_{m, n}
\end{aligned}
$$

Likewise, if (2.7) is multiplied by $x^{m+1} y^{2}$ and then summed for $m=1,2,3, \ldots$, it generates

$$
\begin{aligned}
& (\lambda+2 \nu) \sum_{m=1}^{\infty} x^{m+1} y^{2} \pi_{m, 1}= \\
& \lambda \sum_{m=1}^{\infty} x^{m+1} y^{2} \pi_{m, 0}+\lambda \sum_{m=1}^{\infty} x^{m+1} y^{2} \pi_{m-1,2} \\
& \quad+\nu \sum_{m=1}^{\infty} x^{m+1} y^{2} \pi_{m, 2}+2 \nu \sum_{m=1}^{\infty} x^{m+1} y^{2} \pi_{m+1,0}
\end{aligned}
$$


or,

$$
\begin{aligned}
& (\lambda+2 \nu) x y \sum_{m=1}^{\infty} x^{m} y \pi_{m, 1}= \\
& \lambda x y^{2} \sum_{m=1}^{\infty} x^{m} \pi_{m, 0}+\lambda x^{2} \sum_{m=0}^{\infty} x^{m} y^{2} \pi_{m, 2} \\
& \quad+\nu x \sum_{m=1}^{\infty} x^{m} y^{2} \pi_{m, 2}+2 \nu y^{2} \sum_{m=2}^{\infty} x^{m} \pi_{m, 0}
\end{aligned}
$$

In the same way, multiplying (2.6) by $x^{m+1} y$ and summing through $m=$ $1,2,3, \ldots$, gives

$$
\begin{aligned}
& (\lambda+2 \nu) \sum_{m=1}^{\infty} x^{m+1} y \pi_{m, 0}= \\
& \lambda \sum_{m=1}^{\infty} x^{m+1} y \pi_{m-1,1}+\nu \sum_{m=1}^{\infty} x^{m+1} y \pi_{m, 1}
\end{aligned}
$$

or,

$$
\begin{aligned}
& (\lambda+2 \nu) x y \sum_{m=1}^{\infty} x^{m} \pi_{m, 0}= \\
& \lambda x^{2} \sum_{m=0}^{\infty} x^{m} y \pi_{m, 1}+\nu x \sum_{m=1}^{\infty} x^{m} y \pi_{m, 1}
\end{aligned}
$$

Combining (2.10), (2.12), and (2.14) gives

$$
\begin{aligned}
& (\lambda+2 \nu) x y \sum_{m=1}^{\infty} \sum_{n=0}^{\infty} x^{m} y^{n} \pi_{m, n}= \\
& \lambda x^{2} \sum_{m=0}^{\infty} \sum_{n=1}^{\infty} x^{m} y^{n} \pi_{m, n}+\lambda x y^{2} \sum_{m=1}^{\infty} x^{m} \pi_{m, 0} \\
& \quad+\nu x \sum_{m=1}^{\infty} \sum_{n=1}^{\infty} x^{m} y^{n} \pi_{m, n}+\nu y^{2} \sum_{m=2}^{\infty} \sum_{n=0}^{\infty} x^{m} y^{n} \pi_{m, n} \\
& \quad+\nu y^{2} \sum_{m=2}^{\infty} x^{m} \pi_{m, 0}
\end{aligned}
$$


or,

$$
\begin{aligned}
& (\lambda+2 \nu) x y[P(x, y)-P(0, y)]= \\
& \quad \lambda x^{2}[P(x, y)-P(x, 0)]+\lambda x y^{2}\left[P(x, 0)-\pi_{0,0}\right] \\
& \quad+\nu x\left[P(x, y)-P(x, 0)-P(0, y)+\pi_{0,0}\right] \\
& \quad+\nu y^{2}\left[P(x, y)-P(0, y)-\sum_{n=0}^{\infty} x y^{n} \pi_{1, n}\right] \\
& \quad+\nu y^{2}\left[P(x, 0)-x \pi_{1,0}-\pi_{0,0}\right]
\end{aligned}
$$

Similarly, combining (2.5) and (2.4) gives

$$
\begin{aligned}
& \left(\lambda+\nu^{*}\right) x y \sum_{n=1}^{\infty} y^{n} \pi_{0, n}= \\
& \lambda x y^{2} \pi_{0,0}+\nu y^{2} \sum_{n=0}^{\infty} x y^{n} \pi_{1, n} \\
& \quad+\nu y^{2} x \pi_{1,0}+\nu^{*} x \sum_{n=2}^{\infty} y^{n} \pi_{0, n}
\end{aligned}
$$

or,

$$
\begin{aligned}
& \left(\lambda+\nu^{*}\right) x y\left[P(0, y)-\pi_{0,0}\right]= \\
& \quad \lambda x y^{2} \pi_{0,0}+\nu y^{2} \sum_{n=0}^{\infty} x y^{n} \pi_{1, n} \\
& \quad+\nu y^{2} x \pi_{1,0}+\nu^{*} x\left[P(0, y)-y \pi_{0,1}-\pi_{0,0}\right]
\end{aligned}
$$

Solving (2.18) for $\nu y^{2} \sum_{n=0}^{\infty} x y^{n} \pi_{1, n}$ and substituting the solution into (2.16) gives

$$
\begin{aligned}
& (\lambda+2 \nu) x y[P(x, y)-P(0, y)]= \\
& \quad \lambda x^{2}[P(x, y)-P(x, 0)]+\lambda x y^{2}\left[P(x, 0)-\pi_{0,0}\right] \\
& \quad+\nu x\left[P(x, y)-P(x, 0)-P(0, y)+\pi_{0,0}\right] \\
& \quad+\nu y^{2}[P(x, y)-P(0, y)]+\nu y^{2}\left[P(x, 0)-x \pi_{1,0}-\pi_{0,0}\right] \\
& \quad-\left(\lambda+\nu^{*}\right) x y\left[P(0, y)-\pi_{0,0}\right]+\lambda x y^{2} \pi_{0,0}+\nu y^{2} x \pi_{1,0} \\
& \quad+\nu^{*} x\left[P(0, y)-y \pi_{0,1}-\pi_{0,0}\right]
\end{aligned}
$$


After collecting like terms and using (2.3) to replace $\pi_{0,1}$ with $\pi_{0,0}$, the final form of the functional equation is given as

$$
h_{1}^{*}(x, y) P(x, y)=h_{2}^{*}(x, y) P(x, 0)+h_{3}^{*}(x, y) P(0, y)+h_{4}^{*}(x, y) P(0,0)
$$

where

$$
\begin{aligned}
& h_{1}^{*}(x, y)=(\lambda+2 \nu) x y-\lambda x^{2}-\nu x-\nu y^{2} \\
& h_{2}^{*}(x, y)=\lambda x y^{2}-\lambda x^{2}-\nu x+\nu y^{2} \\
& h_{3}^{*}(x, y)=\left(2 \nu-\nu^{*}\right) x y-\left(\nu-\nu^{*}\right) x-\nu y^{2} \\
& h_{4}^{*}(x, y)=\nu^{*} x y+\left(\nu-\nu^{*}\right) x-\nu y^{2}
\end{aligned}
$$

To simplify the analysis of this model for the purpose of this thesis, the restriction that $\nu^{*}=2 \nu$ is imposed; i.e. when one queue is empty the service rate of the other doubles. The functional equation becomes

$$
h_{1}(x, y) P(x, y)=h_{2}(x, y) P(x, 0)+h_{3}(x, y) P(0, y)+h_{4}(x, y) P(0,0)
$$

where

$$
\begin{aligned}
& h_{1}(x, y)=(\lambda+2 \nu) x y-\lambda x^{2}-\nu x-\nu y^{2} \\
& h_{2}(x, y)=\lambda x y^{2}-\lambda x^{2}-\nu x+\nu y^{2} \\
& h_{3}(x, y)=\nu x-\nu y^{2} \\
& h_{4}(x, y)=2 \nu x y-\nu x-\nu y^{2}
\end{aligned}
$$

The ultimate goal of the subsequent analysis is to explicitly determine $P(x, y)$. This will be done by resolving $P(x, 0)$ and $P(0, y)$ as the solutions to complex boundary value problems.

From an applied perspective an explicit form for $P(x, y)$ is somewhat anticlimactic as some performance measures can be obtained without even fully knowing the characteristic function. This is revealed in the next section.

\subsection{Preliminary Results}

Directly from the functional equation (2.21) significant results are derived. In this section an exact form for $\pi_{0,0}$ which is the long-run probability that both queues are empty is presented. Even the average queue lengths $\mathbb{E} X_{1}=\mathbb{E} X_{2}$ 
are fully derived.

It needs to be noted that these results are easily obtained only because of the very simple and restrictive form of coupling imposed on the model. The first two results depend on finding a function $\gamma(y)$ such that $h_{2}(\gamma(y), y)=$ $h_{3}(\gamma(y), y)=0$. With the simple coupling used it is easy to find that $\gamma(y)=y^{2}$; however, no such function can be found for a more general form of coupling.

\subsection{1 $\pi_{0,0}$}

In this section $\pi_{0,0}$, which is the long-run probability that both queues are empty, is derived.

With $x=\gamma(y)$ such that $h_{2}(\gamma(y), y)=h_{3}(\gamma(y), y)=0$, the functional equation $(2.21)$ reduces to

$$
P(\gamma(y), y)=\frac{h_{4}(\gamma(y), y)}{h_{1}(\gamma(y), y)} P(0,0)
$$

With $\gamma(y)$ found above, $\gamma(1)=1$. As $P(1,1)=1,(2.22)$ can be arranged so that

$$
\begin{aligned}
\pi_{0,0} & =\lim _{y \rightarrow 1} \frac{h_{1}(\gamma(y), y)}{h_{4}(\gamma(y), y)} \\
& =\lim _{y \rightarrow 1} \frac{(\lambda+2 \nu) \gamma(y) y-\lambda(\gamma(y))^{2}-\nu \gamma(y)-\nu y^{2}}{2 \nu \gamma(y) y-\nu \gamma(y)-\nu y^{2}} \\
& =\lim _{y \rightarrow 1} \frac{(\lambda+2 \nu) y-\lambda y^{2}-2 \nu}{2 \nu y-2 \nu} \\
& =\lim _{y \rightarrow 1} \frac{2 \nu(y-1)-\lambda y(y-1)}{2 \nu(y-1)} \\
& =\lim _{y \rightarrow 1} \frac{2 \nu-\lambda y}{2 \nu} \\
& =\frac{2 \nu-\lambda}{2 \nu}
\end{aligned}
$$

From this concise determination of $\pi_{0,0}$ a condition on the existence of $\pi$ can actually be formed. 
Lemma 2.2.1 The network is ergodic if and only if $r<1$ where

$$
r=\frac{\lambda}{2 \nu}
$$

Proof: If the network is ergodic then the stationary distribution $\pi$ exists. If $\pi$ exists, then $0<\pi_{0,0}<1$. Note that $\pi_{0,0}$ can be expressed in terms of $r$ as

$$
\pi_{0,0}=1-r
$$

so, clearly if the network is ergodic then $r<1$.

Intuitively, the coupled JSQ model is more efficient than the uncoupled model. It is well known that the standard JSQ model is ergodic if $r<1$. Logically, then the coupled model will also be ergodic if $r<1$.

\subsubsection{A Relationship between $\mathbb{E} Y_{1}$ and $\mathbb{E} Y_{2}$}

Note that

$$
\begin{aligned}
\frac{\mathrm{d}}{\mathrm{d} y} P(\gamma(y), y)= & \gamma^{\prime}(y) \sum_{m=0}^{\infty} \sum_{n=0}^{\infty} m(\gamma(y))^{m-1} y^{n} \pi_{m, n} \\
& +\sum_{m=0}^{\infty} \sum_{n=0}^{\infty} n(\gamma(y))^{m} y^{n-1} \pi_{m, n}
\end{aligned}
$$

and, therefore,

$$
\left.\frac{\mathrm{d}}{\mathrm{d} y} P(\gamma(y), y)\right|_{y=1}=\gamma^{\prime}(1) \mathbb{E} Y_{1}+\mathbb{E} Y_{2}
$$

From the equation $(2.22), \frac{\mathrm{d}}{\mathrm{d} y} P(\gamma(y), y)$ can be found as

$$
\frac{\mathrm{d}}{\mathrm{d} y} P(\gamma(y), y)=\frac{h_{1}(\gamma(y), y) \frac{\mathrm{d}}{\mathrm{d} y} h_{4}(\gamma(y), y)-h_{4}(\gamma(y), y) \frac{\mathrm{d}}{\mathrm{d} y} h_{1}(\gamma(y), y)}{\left(h_{1}(\gamma(y), y)\right)^{2}} \pi_{0,0}
$$


As $h_{1}(\gamma(y), y)=h_{4}(\gamma(y), y)=0$ l'Hôpital's rule needs to be applied (twice in fact) to compute the limit as $\left.\frac{\mathrm{d}}{\mathrm{d} y} P(\gamma(y), y)\right|_{y=1}$.

First, it is easily found that

$$
\gamma^{\prime}(y)=2 y \quad \gamma^{\prime \prime}(y)=2
$$

Therefore,

$$
\gamma(1)=1, \quad \gamma^{\prime}(1)=2, \quad \gamma^{\prime \prime}(1)=2
$$

Taking the first two derivatives of $h_{1}(\gamma(y), y)$ yields

$$
\begin{aligned}
h_{1}(\gamma(y), y)= & (\lambda+2 \nu) \gamma(y) y-\lambda(\gamma(y))^{2}-\nu \gamma(y)-\nu y^{2} \\
\frac{\mathrm{d}}{\mathrm{d} y} h_{1}(\gamma(y), y)= & (\lambda+2 \nu) \gamma^{\prime}(y) y+(\lambda+2 \nu) \gamma(y) \\
& -2 \lambda \gamma(y) \gamma^{\prime}(y)-\nu \gamma^{\prime}(y)-2 \nu y \\
\frac{\mathrm{d}^{2}}{\mathrm{~d} y^{2}} h_{1}(\gamma(y), y)= & (\lambda+2 \nu) \gamma^{\prime \prime}(y) y+2(\lambda+2 \nu) \gamma^{\prime}(y) \\
& -2 \lambda\left(\gamma^{\prime}(y)\right)^{2}-2 \lambda \gamma(y) \gamma^{\prime \prime}(y)-\nu \gamma^{\prime \prime}(y)-2 \nu
\end{aligned}
$$

So,

$$
\left.\frac{\mathrm{d}}{\mathrm{d} y} h_{1}(\gamma(y), y)\right|_{y=1}=2 \nu-\lambda,\left.\quad \frac{\mathrm{d}^{2}}{\mathrm{~d} y^{2}} h_{1}(\gamma(y), y)\right|_{y=1}=8 \nu-6 \lambda
$$

Likewise, for $h_{4}(\gamma(y), y)$

$$
\begin{aligned}
h_{4}(\gamma(y), y) & =2 \nu \gamma(y) y-\nu \gamma(y)-\nu y^{2} \\
\frac{\mathrm{d}}{\mathrm{d} y} h_{4}(\gamma(y), y) & =2 \nu \gamma^{\prime}(y) y+2 \nu \gamma(y)-\nu \gamma^{\prime}(y)-2 \nu y \\
\frac{\mathrm{d}^{2}}{\mathrm{~d} y^{2}} h_{4}(\gamma(y), y) & =2 \nu \gamma^{\prime \prime}(y) y+4 \nu \gamma^{\prime}(y)-\nu \gamma^{\prime \prime}(y)-2 \nu
\end{aligned}
$$

and

$$
\left.\frac{\mathrm{d}}{\mathrm{d} y} h_{4}(\gamma(y), y)\right|_{y=1}=2 \nu,\left.\quad \frac{\mathrm{d}^{2}}{\mathrm{~d} y^{2}} h_{4}(\gamma(y), y)\right|_{y=1}=8 \nu
$$


Therefore,

$$
\begin{aligned}
\left.\frac{\mathrm{d}}{\mathrm{d} y} P(\gamma(y), y)\right|_{y=1} & =\lim _{y \rightarrow 1} \frac{h_{1}(\gamma(y), y) \frac{\mathrm{d}}{\mathrm{d} y} h_{4}(\gamma(y), y)-h_{4}(\gamma(y), y) \frac{\mathrm{d}}{\mathrm{d} y} h_{1}(\gamma(y), y)}{\left(h_{1}(\gamma(y), y)\right)^{2}} \pi_{0,0} \\
& =\lim _{y \rightarrow 1} \frac{h_{1}(\gamma(y), y) \frac{\mathrm{d}^{2}}{\mathrm{~d} y^{2}} h_{4}(\gamma(y), y)-h_{4}(\gamma(y), y) \frac{\mathrm{d}^{2}}{\mathrm{~d} y^{2}} h_{1}(\gamma(y), y)}{2 h_{1}(\gamma(y), y) \frac{\mathrm{d}}{\mathrm{d} y} h_{1}(\gamma(y), y)} \pi_{0,0} \\
& =\lim _{y \rightarrow 1} \frac{\frac{-\frac{\mathrm{d}}{\mathrm{d} y} h_{1}(\gamma(y), y) \frac{\mathrm{d}^{2}}{\mathrm{~d} y^{2}} h_{4}(\gamma(y), y) \frac{\mathrm{d}^{2}}{\mathrm{~d} y^{2}} h_{1}(\gamma(y), y)-h_{1}(\gamma(y), y) \frac{\mathrm{d}^{3}}{\mathrm{~d} y^{3}} h_{4}(\gamma(y), y)}{2\left(\frac{\mathrm{d}}{\mathrm{d} y} h_{1}(\gamma(y), y)\right)^{2}+2 h_{1}(\gamma(y), y) \frac{\mathrm{d}^{2}}{\mathrm{~d} y^{2}} h_{1}(\gamma(y), y)} h_{1}(\gamma(y), y)}{\pi_{0,0}} \pi_{0,0} \\
& \left.=\frac{2\left(\left.\left.\frac{\mathrm{d}}{\mathrm{d} y} h_{1}(\gamma(y), y)\right|_{y=1} \frac{\mathrm{d}^{2}}{\mathrm{~d} y^{2}} h_{4}(\gamma(y), y)\right|_{y=1}\right.}{\left.-\left.\frac{\mathrm{d}}{\mathrm{d} y} h_{1}(\gamma(y), y)\right|_{y=1}\right)\left.\left.^{2}(\gamma(y), y)\right|_{y=1} \frac{\mathrm{d}^{2}}{\mathrm{~d} y^{2}} h_{1}(\gamma(y), y)\right|_{y=1}}\right] \\
& =\frac{2 \lambda \nu}{(2 \nu-\lambda)^{2}}=\frac{r}{(1-r)^{2}}
\end{aligned}
$$

In summary,

Lemma 2.2.2 $\mathbb{E} Y_{1}$ and $\mathbb{E} Y_{2}$ are related as

$$
2 \mathbb{E} Y_{1}+\mathbb{E} Y_{2}=\frac{r}{(1-r)^{2}}
$$

Because of this relationship, only one of $\mathbb{E} Y_{1}$ and $\mathbb{E} Y_{2}$ needs to be calculated to have the other.

\subsubsection{Individual Forms for $\mathbb{E} Y_{1}$ and $\mathbb{E} Y_{2}$}

The preliminary principles required to compute $\mathbb{E} Y_{1}$ and $\mathbb{E} Y_{2}$ individually are now outlined; however, the solution to the complex boundary value problem is required to complete the result.

First, a relation between $P(0,1)$ and $P(1,0)$ can be revealed. If $y=x$, the 
functional equation (2.21) becomes

$$
\begin{aligned}
& h_{1}(x, x)=x(x-1) \nu \\
& h_{2}(x, x)=x(x-1)(\lambda x+\nu) \\
& h_{3}(x, x)=-x(x-1) \nu \\
& h_{4}(x, x)=x(x-1) \nu
\end{aligned}
$$

The common factor $x(x-1)$ can be removed to reveal

$$
\nu P(x, x)=(\lambda x+\nu) P(x, 0)-\nu P(0, x)+\nu \pi_{0,0}
$$

Setting $x=1$ yields

$$
(\lambda+\nu) P(1,0)-\nu P(0,1)=\nu\left(1-\pi_{0,0}\right)
$$

which can be simplified using (2.28) to

\section{Lemma 2.2.3}

$$
(\lambda+\nu) P(1,0)-\nu P(0,1)=\frac{\lambda}{2}
$$

This result will be used a bit later.

Taking the derivative of the functional equation (2.21) with respect to $y$ after setting $x=1$ allows $\mathbb{E} Y_{2}$ to be computed as

$$
\mathbb{E} Y_{2}=\left[\frac{\mathrm{d}}{\mathrm{d} y} P(1, y)\right]_{y=1}
$$

In the functional equation (2.21), letting $x=1$ gives

$$
\begin{aligned}
& h_{1}(1, y)=(y-1)[\lambda-\nu(y-1)] \\
& h_{2}(1, y)=(y-1)(y+1)(\lambda+\nu) \\
& h_{3}(1, y)=-(y-1)(y+1) \nu \\
& h_{4}(1, y)=-(y-1)^{2} \nu
\end{aligned}
$$

After dividing through by the common factor $(y-1)$, the functional equation becomes

$$
[\lambda-\nu(y-1)] P(1, y)=(y+1)(\lambda+\nu) P(1,0)-(y+1) \nu P(0, y)-(y-1) \nu \pi_{0,0}
$$


Taking the derivative with respect to $y$ gives

$$
\begin{aligned}
& -\nu P(1, y)+[\lambda-(y-1) \nu] \frac{\mathrm{d}}{\mathrm{d} y} P(1, y)= \\
& \quad(\lambda+\nu) P(1,0)-\nu P(0, y)-(y+1) \nu \frac{\mathrm{d}}{\mathrm{d} y} P(0, y)-\nu \pi_{0,0}
\end{aligned}
$$

Setting $y=1$ yields

$$
\begin{aligned}
& -\nu+\lambda \mathbb{E} Y_{2}= \\
& \quad(\lambda+\nu) P(1,0)-\nu P(0,1)-\left.2 \nu \frac{\mathrm{d}}{\mathrm{d} y} P(0, y)\right|_{y=1}-\nu \pi_{0,0}
\end{aligned}
$$

This expression can be simplified using the relationship between $P(0,1)$ and $P(1,0)$ established above $(2.52)$ and $\pi_{0,0}$ as in (2.28) to obtain

\section{Lemma 2.2.4}

$$
\mathbb{E} Y_{2}=1-\left.\frac{1}{r} \frac{d}{d y} P(0, y)\right|_{y=1}
$$

In the next section it is shown how $P(0, y)$ and its derivative can be computed as the solution to a complex boundary value problem.

In a similar manner a form for $\mathbb{E} Y_{1}$ can be derived. This time, letting $y=1$ in the functional equation (2.21), gives

$$
\begin{aligned}
& h_{1}(x, 1)=-(x-1)(\lambda x-\nu) \\
& h_{2}(x, 1)=-(x-1)(\lambda x+\nu) \\
& h_{3}(x, 1)=(x-1) \nu \\
& h_{4}(x, 1)=(x-1) \nu
\end{aligned}
$$

And dividing through by $(x-1)$ yields

$$
-(\lambda x-\nu) P(x, 1)=-(\lambda x+\nu) P(x, 0)+\nu P(0,1)+\nu \pi_{0,0}
$$

Taking the derivative with respect to $x$ gives

$$
-\lambda P(x, 1)-(\lambda x-\nu) \frac{\mathrm{d}}{\mathrm{d} x} P(x, 1)=-\lambda P(x, 0)-(\lambda x+\nu) \frac{\mathrm{d}}{\mathrm{d} x} P(x, 0)
$$


Finally, setting $x=1$ yields

$$
\left(r-\frac{1}{2}\right) \mathbb{E} Y_{1}=r P(1,0)-r+\left.\left(r+\frac{1}{2}\right) \frac{\mathrm{d}}{\mathrm{d} x} P(x, 0)\right|_{x=1}
$$

So,

Lemma 2.2.5 For $r \neq \frac{1}{2}$ (i.e. $\lambda \neq \nu$ )

$$
\mathbb{E} Y_{1}=\frac{1}{r-\frac{1}{2}}\left[r P(1,0)-r+\left.\left(r+\frac{1}{2}\right) \frac{d}{d x} P(x, 0)\right|_{x=1}\right]
$$

Both $P(1,0)$ and $\left.\frac{\mathrm{d}}{\mathrm{d} x} P(x, 0)\right|_{x=1}$ can be computed from the solution to the complex boundary value problem as presented in the next section. Note though that if $\mathbb{E} Y_{2}$ is know, then $\mathbb{E} Y_{1}$ can be determined from (2.45) even if $\lambda=\nu$.

While knowing $\mathbb{E} Y_{1}$ and $\mathbb{E} Y_{2}$ is interesting, the average queue lengths $\mathbb{E} X_{1}=$ $\mathbb{E} X_{2}$ is the most commonly sought performance measures.

\section{Lemma 2.2.6}

$$
\mathbb{E} X_{1}=\mathbb{E} X_{2}=\frac{r}{2(1-r)^{2}}
$$

Proof: It should be clear that

$$
\mathbb{E}\left\{\min \left(X_{1}, X_{2}\right)\right\}=\mathbb{E} Y_{1}
$$

and

$$
\mathbb{E}\left\{\max \left(X_{1}, X_{2}\right)\right\}=\mathbb{E}\left\{\min \left(X_{1}, X_{2}\right)\right\}+\mathbb{E}\left|X_{1}-X_{2}\right|=\mathbb{E} Y_{1}+\mathbb{E} Y_{2}
$$

Therefore, the expected individual queue lengths should just be the average of the expected maximum and minimum, or,

$$
\mathbb{E} X_{1}=\mathbb{E} X_{2}=\frac{1}{2}\left\{2 \mathbb{E} Y_{1}+\mathbb{E} Y_{2}\right\}
$$

The lemma follows from (2.45).

The determination of $\mathbb{E} Y_{1}$ and $\mathbb{E} Y_{2}$ depends on the functions $P(x, 0)$ and 
$P(0, y)$ respectively, which can only be numerically evaluated based on a boundary value problem solution. However, the explicit determination of the average queue lengths $\mathbb{E} X_{1}$ and $\mathbb{E} X_{2}$ is achieved simply by exploiting a relationship between $\mathbb{E} Y_{1}$ and $\mathbb{E} Y_{2}$ derived directly from the functional equation.

Proceeding in the analysis of $P(x, y)$ may seem somewhat pointless now that have determined the average queue lengths. However, it needs to be remembered that this result is easily obtained only because the service coupling is so simple. Considering a more general form of coupling or even a JSQ model in which each queue has its own service rate (i.e. $\nu_{1} \neq \nu_{2}$ ) would require the determination of both $P(0, y)$ and $P(x, 0)$ to provide any meaningful results. Also, the mean queue lengths is not the only useful result, for example, determination of the tail asymptotics is a vital aid to simulation. So, for the sake of further research the following sections are presented. Besides, life isn't always about reaching a destination. The journey can be just as enjoyable: a principle I've tried to keep in mind while laboring on this thesis.

\subsection{A Boundary Value Problem Solution for $P(0, y)$}

To describe $P(x, y)$ explicitly, both $P(x, 0)$ and $P(0, y)$ need to be determined. First considered is $P(0, y)$. Why? Well, the computation is slightly simpler than for $P(x, 0)$ and the result is more complete; also, this is the order in which van Leeuwaarden [11] proceeds.

In a nutshell, the procedure of analysis is to first select a closed contour from the zero-set of the kernel $h_{1}(x, y)$ from the functional equation (2.21). The value of $P(0, y)$ on this contour is found. After showing that $P(0, y)$ is continous on and within this contour and it is analytic within the region bounded by the contour, a boundary value problem is solved to give $P(0, y)$ on and within the contour.

\subsubsection{Analysis of the Kernel}

The zeros of the kernel $h_{1}(x, y)$, are characterized in this section. $h_{1}(x, y)$ can be viewed as a degree-2 polynomial in $y$. For every value of $x$, therefore, there are two possible values, say $y_{+}(x)$ and $y_{-}(x)$, such that $h_{1}\left(x, y_{+}(x)\right)=$ 
$h_{1}\left(x, y_{-}(x)\right)=0$.

Using the definition $r=\frac{\lambda}{2 \nu}$, then $h_{1}(x, y)=0$ is equivalent to,

$$
y^{2}-2(r+1) x y+(2 r x+1) x=0
$$

The zeros of the kernel are given as

$$
\begin{aligned}
& y_{+}(x)=(r+1) x+\sqrt{\left(r^{2}+1\right) x^{2}-x} \\
& y_{-}(x)=(r+1) x-\sqrt{\left(r^{2}+1\right) x^{2}-x}
\end{aligned}
$$

or more concisely, by the two valued function

$$
y(x)=(r+1) x \pm \sqrt{\left(r^{2}+1\right) x^{2}-x}
$$

The zero-space of $h_{1}(x, y)$ is therefore $\{(x, y): x \in \mathbb{C}, y=y(x)\}$. Likewise, $h_{1}(x, y)$ can also be viewed as a polynomial in $x$ of degree 2 . So, $h_{1}(x, y)=0$ is equivalent to

$$
2 r x^{2}+[1-2(r+1) y] x+y^{2}=0
$$

For every value of $y$ there are two values of $x$, say $x_{+}(y)$ and $x_{-}(y)$ such that $h_{1}\left(x_{+}(y), y\right)=h_{1}\left(x_{-}(y), y\right)=0$. These values are given as

$$
\begin{aligned}
& x_{+}(y)=\frac{2(r+1) y-1+\sqrt{[2(r+1) y-1]^{2}-8 r y^{2}}}{4 r} \\
& x_{-}(y)=\frac{2(r+1) y-1-\sqrt{[2(r+1) y-1]^{2}-8 r y^{2}}}{4 r}
\end{aligned}
$$

Again, the two valued function

$$
x(y)=\frac{2(r+1) y-1 \pm \sqrt{[2(r+1) y-1]^{2}-8 r y^{2}}}{4 r}
$$

can be defined. So, the zero-space of $h_{1}(x, y)$ can also be expressed as $\{(x, y): y \in \mathbb{C}, x=x(y)\}$

The following lemmas describe the algebraic function $y(x)$. When providing a solution for $P(x, 0)$ in the next section, the same lemmas are developed for $x(y)$. 


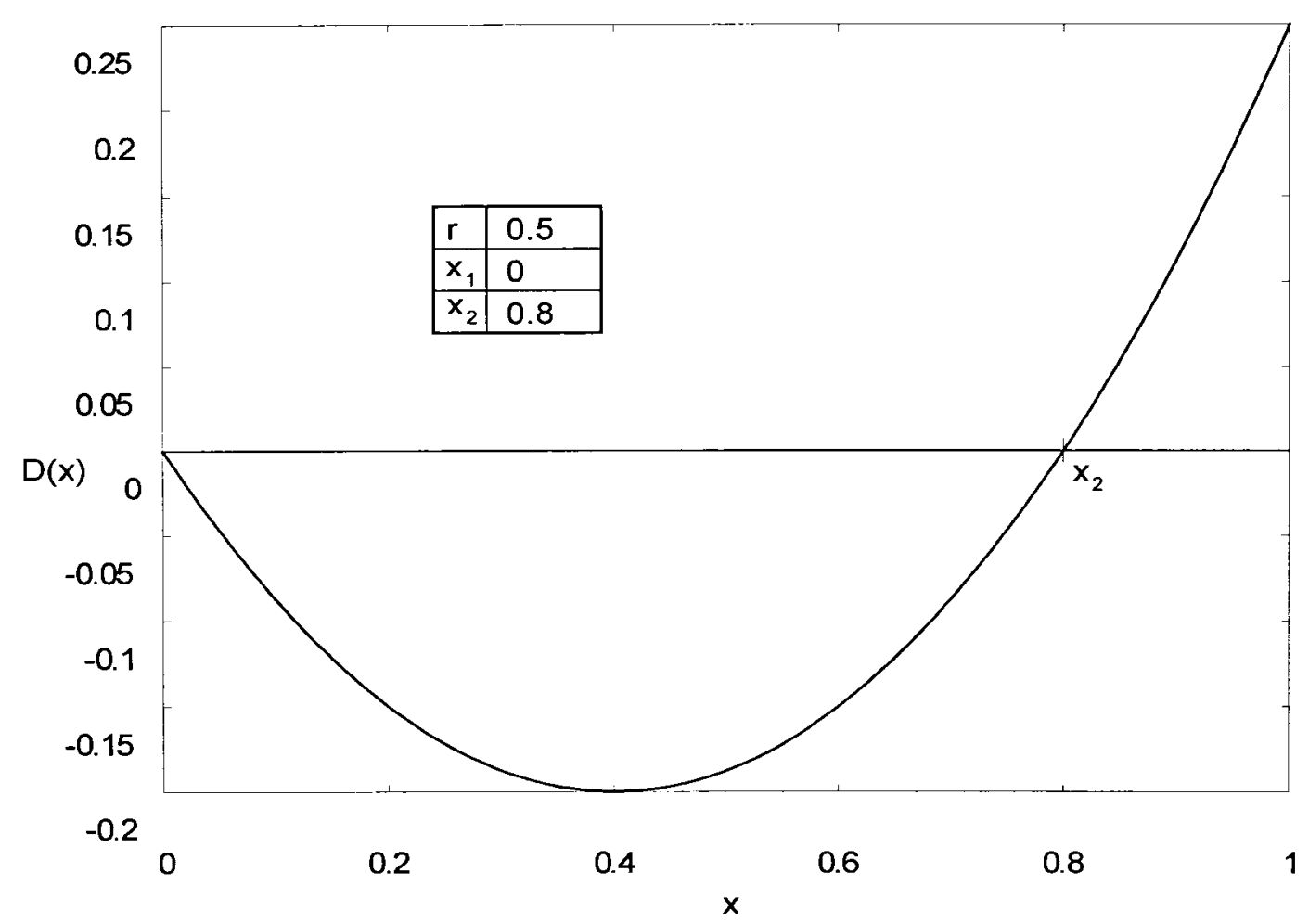

Figure 2.3: Coupled - JSQ: Discriminant as a Function of Real $x$

Lemma 2.3.1 The algebraic function $y(x)$ defined by $h_{1}(x, y(x))=0$, has two real branch points $0=x_{1}<x_{2}<1$.

Proof: The branch points of $y(x)$ are the points where the derivative with respect to $x$ is undefined. Equivalently, they are the zeros of the discriminant, which is

$$
D_{1}(x)=\left(r^{2}+1\right) x^{2}-x
$$

$D_{1}(x)=0$ at $x_{1}=0$ and at $x_{2}=\frac{1}{r^{2}+1}$. As $r>0$ it must be true that $0=x_{1}<x_{2}<1$.

An appropriate sub-set of the kcrncl's zero-space is now selected. Specifically, $x$ is restricted to be real and in the interval $\left[x_{1}, x_{2}\right]$. Figure 2.3 graphs the discriminant of $y(x)$ for real valued $x$ between 0 and 1 . 
Lemma 2.3.2 For $x \in\left[x_{1}, x_{2}\right], y(x)$ lies on the closed contour $L$, which is symmetric with respect to the real line, and defined by

$$
|y|^{2}=m(R e(y))
$$

where

$$
m(\delta)=(2 r \zeta(\delta)+1) \zeta(\delta)
$$

and

$$
\zeta(\delta)=\frac{\delta}{r+1}
$$

It further holds that

$$
|y| \leq \alpha
$$

where

$$
\alpha=\frac{r+1}{r^{2}+1}
$$

Proof: Note that

$$
D_{1}\left(\frac{x_{2}}{2}\right)=\frac{1}{4\left(r^{2}+1\right)}-\frac{1}{2\left(r^{2}+1\right)}<0
$$

So, for all $x \in\left(x_{1}, x_{2}\right), D_{1}(x)$ is negative. This is illustrated nicely in Figure 2.3. As $D_{1}(x)$ is negative then $y(x)$ is complex. Naturally, $y_{+}(x)$ and $y_{-}(x)$ are complex conjugates. Therefore,

$$
\operatorname{Re}(y(x))=(r+1) x
$$

and

$$
\operatorname{Im}(y(x))= \pm \sqrt{x-\left(r^{2}+1\right) x^{2}}
$$

So,

$$
|y(x)|^{2}=(2 r x+1) x
$$

and

$$
|y(x)|=\sqrt{(2 r x+1) x}
$$




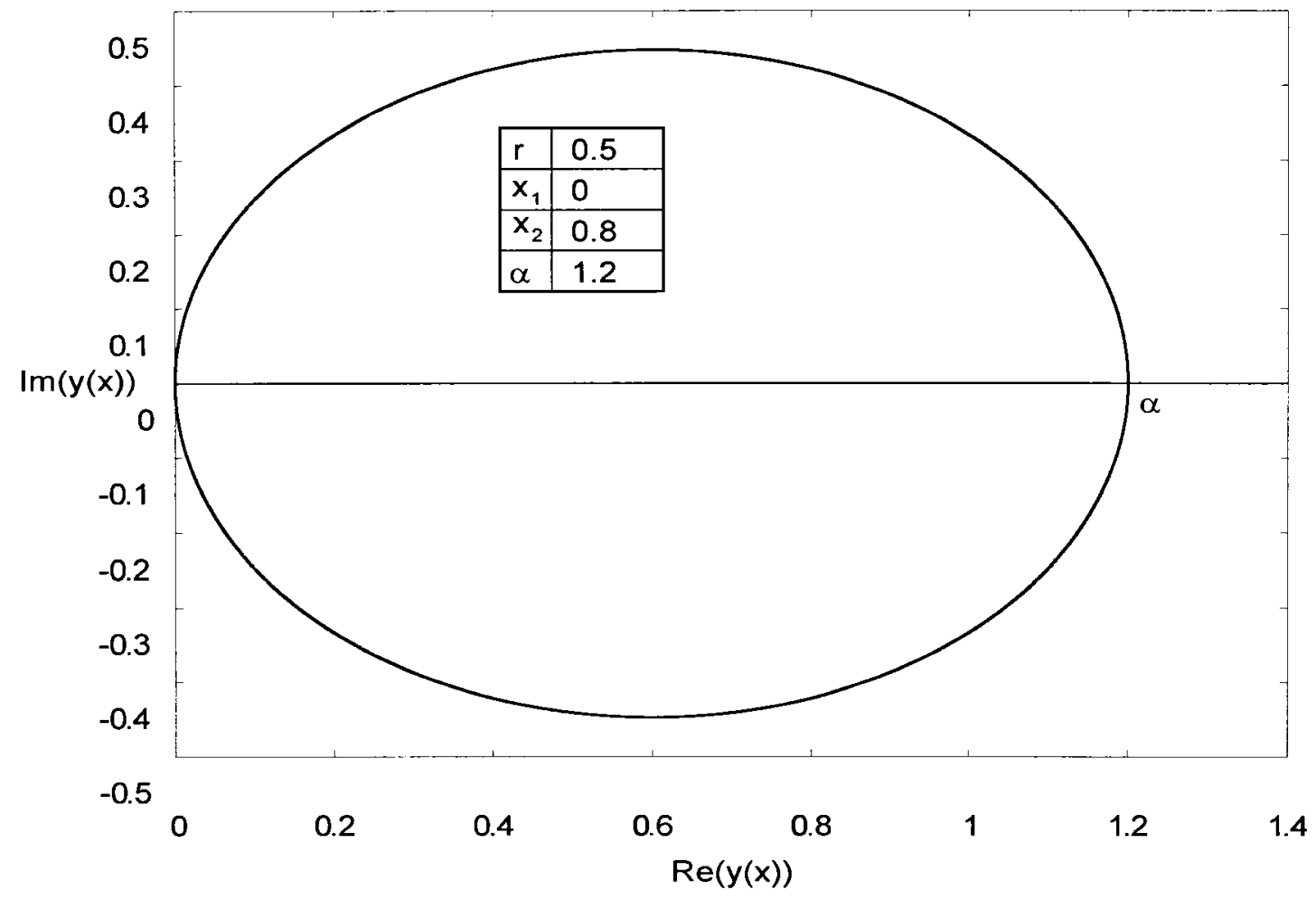

Figure 2.4: Coupled - JSQ: The Contour $L$

Clearly $|y(x)|$ is increasing in $x$. So,

$$
|y(x)| \leq\left|y\left(x_{2}\right)\right|=\frac{r+1}{r^{2}+1}=\alpha
$$

Finally, $\zeta(\delta)$ is derived by solving (2.89) for $x$ with $\delta=\operatorname{Re}(y(x))$. This function allows the definition of $L$ using $y$ alone.

Figure 2.4 illustrates the contour $L$ for a specific value of $r . y(x)$ is plotted in the complex plane for real $x \in\left[x_{1}, x_{2}\right]$.

Corollary 2.3.1 Let $L^{+}$be the interior of the contour $L$. The points $y=0$ and $y=1$ both lie in $L \cup L^{+}$. 
Proof: As $x_{1}=0$, it is clear that $y(0)=y\left(x_{1}\right)=0$. So, $0 \in L$.

Taking the derivative of $\alpha$ with respect to $r$ gives

$$
\frac{\mathrm{d} \alpha}{\mathrm{d} r}=-\frac{r^{2}+2 r-1}{\left(r^{2}+1\right)^{2}}
$$

Now, for $\frac{\mathrm{d} \alpha}{\mathrm{d} r}=0$ it requires $r=-1 \pm \sqrt{2}$. Also, $\left.\frac{\mathrm{d} \alpha}{\mathrm{d} r}\right|_{r=0}>0$ and $\left.\frac{\mathrm{d} \alpha}{\mathrm{d} r}\right|_{r=1}<0$; so, $\alpha$ is increasing for $0<r<-1+\sqrt{2}$ and decreasing for $r>-1+\sqrt{2}$. This means,

$$
\alpha>\left.\alpha\right|_{r=0}=1
$$

and

$$
\alpha \leq\left.\alpha\right|_{r=-1+\sqrt{2}} \approx 1.207
$$

So, the curve $L$ is not contained within the unit circle. For $r>0$, it is always true that $1 \in L^{+}$.

In Figure 2.4 it is clear that $y=0$ is on the contour and that $y=1$ is within the region bounded by it.

\subsubsection{Analytic Continuation}

A common way of defining functions in complex analysis is to first specify the function on a small domain only, and then extend it by analytic continuation. In this thesis, $P(0, y)$, as first defined in $(1.1)$, is analytic only within the unit circle. The goal of this section is to analytically continue $P(0, y)$ from the unit circle to the region bounded by the contour $L$.

Analytic continuation can be defined as follows:

Let $f$ be an analytic function defined on an open subset $U$ of a complex space. If $V$ is a larger open subset, containing $U$, and $F$ is an analytic function defined on $V$ such that $F(z)=f(z)$ for all $z$ in $U$ then $F$ is called an analytic continuation of $f$. If $V$ is connected then $F$ is the unique analytic continuation of $f$ on $V$.

The analytic procedure used thus far is very similar to that used in [1], [11], and [13]. However, the method of analytic continuation used in these papers 
seems to skip some crucial details. An alternate development is therefore introduced in this thesis. The claim is identical, namely that $P(0, y)$ is analytic on $L^{+}$; however, the proof is different.

Before proceeding to the analytic continuation, the following result is required.

Conjecture 2.3.1 For each $y \in L^{+}$there exists an $x$ such that $|x| \leq 1$ and $y=y(x)$. This $x$ is given by one of $x_{+}(y)$ or $x_{-}(y)$

For $y \in L$ no such conjecture is required as the contour $L$ is formed by such pairings. However, for $y \in L^{+}$a rigorous proof is required to support the conjecture. Through numerical observation, the conjecture appears true.

Lemma 2.3.3 The function $P(0, y)$ is analytic in the domain $L^{+}$.

Proof: For $|y|<1, P(0, y)$ is analytic by definition. For zero-pairs $(x, y)$ of the kernel $h_{1}(x, y)$ such that $P(x, y)$ is finite, it is true that

$$
\begin{aligned}
P(0, y) & =-\frac{h_{2}(x, y)}{h_{3}(x, y)} P(x, 0)-\frac{h_{4}(x, y)}{h_{3}(x, y)} P(0,0) \\
& =(2 r x+1) P(x, 0)-\frac{2 x y-x-y^{2}}{x-y^{2}} P(0,0)
\end{aligned}
$$

The term $\left(x-y^{2}\right)=0$ only for the real tuples $(x, y)=(0,0),(1,1),\left(\frac{1}{r^{2}}, \frac{1}{r}\right)$. The first two are also zeros of the numerator and happen to be removable. For the third, as $0<r<1, x=\frac{1}{r^{2}}$ is outside the unit circle and $y=\frac{1}{r}>\alpha=\frac{r+1}{r^{2}+1}$ so it is not in $L^{+}$.

Therefore, for $y \in L^{+}$such that $|y|<1$ and $x=x(y)$ as per the above conjecture, the right hand side of $(2.97)$ is analytic as $P(x, 0)$ is analytic for $|x|<1$ and the second term is non-singular.

Now, if the restriction that $|y|<1$ is removed, the right hand side of (2.97) continues to be analytic for all $y \in L^{+}$and it provides the unique analytic continuation of $P(0, y)$ to $L^{+}$.

Corollary 2.3.2 For $y \in L, P(0, y)$ satisfies the condition

$$
\operatorname{Im}[P(0, y)]=\operatorname{Im}\left[-\frac{2 \eta(y) y-\eta(y)-y^{2}}{\eta(y)-y^{2}}\right] P(0,0)
$$


where

$$
\eta(y)=\frac{\sqrt{1+8 r|y|^{2}}-1}{4 r}
$$

Proof: According to the way $L$ is defined, for $y \in L, x=x(y)$ is real. The relation $(2.98)$ is true because $(2 r x+1) P(x, 0)$, in 2.97 , is strictly real for real $x$.

The function $\eta(y)$ comes from solving (2.91) for $x \in\left[x_{1}, x_{2}\right]$. The boundary condition may be described in terms of $y$ alone by means of $\eta(y)$.

On the contour $L$, therefore, $P(0, y)$ is finite and within the contour it is analytic. The values of $P(0, y)$ on this contour can be expressed as a function of $y$ alone through (2.98). These are the necessary conditions to find $P(0, y)$ as the solution to a boundary value problem.

\subsubsection{The Boundary Value Problem}

The determination of $P(0, y)$ reduces to the solution of a Riemann-Hilbert boundary value problem on the contour $L$. The theory behind the following statements is fully developed in Cohen \& Boxma's text [2] section I.3.

A determination of $P(0, y)$ needs to be made such that

1. $P(0, y)$ is analytic for $y \in L^{+}$and continuous for $y \in L \cup L^{+}$

2. $\operatorname{Re}[i P(0, y)]=c(y)$ for $y \in L$ where

$$
c(y)=-\operatorname{Im}\left[\frac{2 \eta(y) y-\eta(y)-y^{2}}{\eta(y)-y^{2}}\right] P(0,0)
$$

The standard way to solve this type of boundary value problem is to transform the boundary condition of $(2.98)$ to a condition on the unit circle. The conformal mapping

$$
z=f(y): L^{+} \rightarrow C^{+}
$$

and its inverse

$$
y=f_{0}(z): C^{+} \rightarrow L^{+}
$$

are introduced, where $C^{+}$is the interior of the unit circle $C$. Using these mappings the Riemann-Hilbert problem on $L$ reduces to a Dirichlet problem 
on the unit circle. The solution to this problem gives

$$
P(0, y)=-\frac{1}{2 \pi} \oint_{C} c\left(f_{0}(w)\right) \frac{w+f(y)}{w-f(y)} \frac{\mathrm{d} w}{w}+\kappa_{1}, \quad y \in L \cup L^{+}
$$

where $\kappa_{1}$ is a constant. As $P(0,0)=\pi_{0,0}$ is known from $(2.28), \kappa_{1}$ can be determined by evaluating (2.100) with $y=0$.

To compute $\mathbb{E} Y_{2}$, the derivative of $P(0, y)$ is needed. It is given by

$$
\frac{\mathrm{d}}{\mathrm{d} y} P(0, y)=-\frac{1}{\pi} \oint_{C} c\left(f_{0}(w)\right) \frac{f^{\prime}(y)}{(w-f(y))^{2}} \mathrm{~d} w, \quad y \in L \cup L^{+}
$$

To proceed, $f, f^{\prime}$ and $f_{0}$ need to be determined.

\subsubsection{Determination of the Conformal Mapping and its Inverse}

It may be possible to determine an explicit form for $f$. Perhaps, such a mapping is presented in a dictionary of conformal maps such as [10].

In this thesis, however, instead of presenting an explicit form for the mapping $f$, a procedure is outlined to determine the value of the mapping and its inverse at specific values. This procedure is general enough to apply to all the boundary value problems we describe in the thesis. This procedure, as described in detail in Cohen \& Boxma [2] section I.4, allows the computation of $P(0, y)$ and $\frac{\mathrm{d}}{\mathrm{d} y} P(0, y)$ for specific values of $y \in L \cup L^{+}$as is shown below.

First, $L$ can be represented in polar coordinates as

$$
L=\left\{y: y=\rho(\theta) e^{i \theta}, \quad 0 \leq \theta \leq 2 \pi\right\}
$$

Since $0 \in L \cup L^{+}$, for each point on $L$, the relation between its absolute value and its real part is given by $|y|^{2}=m(\operatorname{Re}(y))$, where $m$ is defined in (2.84). Given the angle $\theta$ of some point on $L$, the real part of this point, to be denoted by $\delta(\theta)$, is the solution of

$$
\delta-\cos \theta \sqrt{m(\delta)}=0, \quad 0 \leq \theta \leq 2 \pi
$$

As $L$ is a smooth, egg-shaped contour, the solution is unique. It is clear that $\rho(\theta)=\frac{\delta(\theta)}{\cos \theta}$, and the parametrization of $L$ is fully specified.

Figure 2.5 illustrates the mapping between $L$ and the unit circle. 


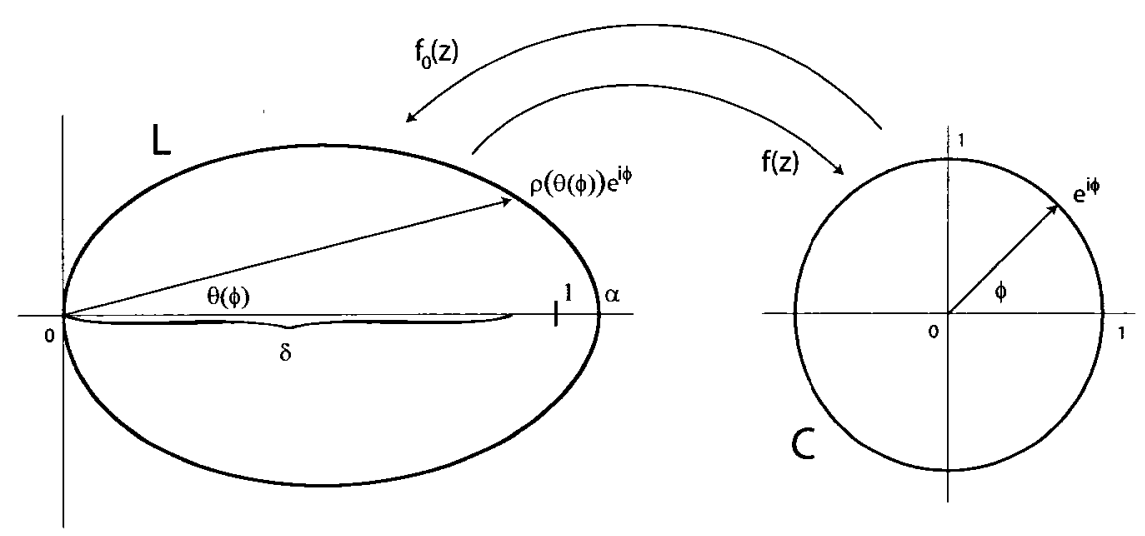

Figure 2.5: Coupled - JSQ: The Conformal Mapping to the Unit Circle

For a contour, such as $L$, that can be described in polar coordinates, the mapping from $z \in C^{+}$to $y \in L^{+}$, where $z=e^{i \phi}$ and $y=\rho(\theta) e^{i \theta}$, is formally given by

$$
f_{0}(z)=z \exp \left[\frac{1}{2 \pi} \int_{0}^{2 \pi} \log \{\rho(\theta(\omega))\} \frac{e^{i \omega}+z}{e^{i \omega}-z} \mathrm{~d} \omega\right], \quad|z|<1
$$

with the angular deformation $\theta$ uniquely determined as the solution of Theodorsen's integral equation

$$
\theta(\phi)=\phi-\int_{0}^{2 \pi} \log \{\rho(\theta(\omega))\} \cot \left\{\frac{1}{2}(\omega-\phi)\right\} d \omega, \quad 0 \leq \phi \leq 2 \pi
$$

This integral equation is best solved iteratively. Starting with $\theta_{0}=\phi$ then taking, for $k=0,1,2,3, \ldots$,

$$
\theta_{k+1}(\phi)=\phi-\int_{0}^{2 \pi} \log \left\{\frac{\delta\left(\theta_{k}(\omega)\right)}{\cos \left(\theta_{k}(\omega)\right)}\right\} \cot \left\{\frac{1}{2}(\omega-\phi)\right\} \mathrm{d} \omega, \quad 0 \leq \phi \leq 2 \pi
$$

gives $\lim _{k \rightarrow \infty} \theta_{k+1}(\phi)=\theta(\phi)$. From (2.102) it is seen that $\delta\left(\theta_{k}(\omega)\right)$ is determined as the value $d$ such that

$$
d-\cos \theta_{k}(\omega) \sqrt{m(d)}=0
$$


which can be solved using a numerical root finding mechanism such as the Newton-Rhapson method.

Finally, $f(y)$ for $y \in L \cup L^{+}$is obtained as the unique solution $z$ of $f_{0}(z)=y$. The contour integrals for $P(0, y)$ and $\frac{\mathrm{d}}{\mathrm{d} y} P(0, y)$ can be rewritten as integrals along the real line if the substitution $w=e^{i \phi}$ is made. Respectively, these computationally friendly forms are given as

$$
P(0, y)=-\frac{i}{2 \pi} \int_{0}^{2 \pi} c\left(f_{0}\left(e^{i \phi}\right)\right) \frac{e^{i \phi}+f(y)}{e^{i \phi}-f(y)} \mathrm{d} \phi+\kappa_{1} \quad y \in L \cup L^{+}
$$

and

$$
\frac{\mathrm{d}}{\mathrm{d} y} P(0, y)=-\frac{i}{\pi} \int_{0}^{2 \pi} c\left(f_{0}\left(e^{i \phi}\right)\right) \frac{f^{\prime}(y)}{\left(e^{i \phi}-f(y)\right)^{2}} \mathrm{~d} \phi \quad y \in L \cup L^{+}
$$

A full solution for $P(0, y)$ with $y \in L \cup L^{+}$has thus been provided.

Computing $\mathbb{E} Y_{2}$ requires the evaluation of $P(0, y)$ and its derivative at $y=1$. As concluded in Corollary 2.3.1, $1 \in L^{+}$; so, it is permissible to compute

$$
P(0,1)=-\frac{i}{2 \pi} \int_{0}^{2 \pi} c\left(f_{0}\left(e^{i \phi}\right)\right) \frac{e^{i \phi}+f(1)}{e^{i \phi}-f(1)} \mathrm{d} \phi+\kappa_{1}
$$

and

$$
\left.\frac{\mathrm{d}}{\mathrm{d} y} P(0, y)\right|_{y=1}=-\frac{i}{\pi} \int_{0}^{2 \pi} c\left(f_{0}\left(e^{i \phi}\right)\right) \frac{f^{\prime}(1)}{\left(e^{i \phi}-f(1)\right)^{2}} \mathrm{~d} \phi
$$

where $f(1)$ is the unique solution to $f_{0}(z)=1$ and

$$
f^{\prime}(1)=\left[\frac{1}{f(1)}+\frac{1}{2 \pi} \exp \left[\frac{1}{2 \pi} \int_{0}^{2 \pi} \log \left\{\frac{\delta(\theta(\omega))}{\cos (\theta(\omega))}\right\} \frac{2 e^{i \omega}}{\left(e^{i \omega}-f(1)\right)^{2}} \mathrm{~d} \omega\right]\right]^{-1}
$$

$\theta(\omega)$ must be found as the solution Theodorsen's integral equation.

The ability to compute $\mathbb{E} Y_{2}$ also allows the computation of $\mathbb{E} Y_{1}$ using the relationship between the two (2.45). 


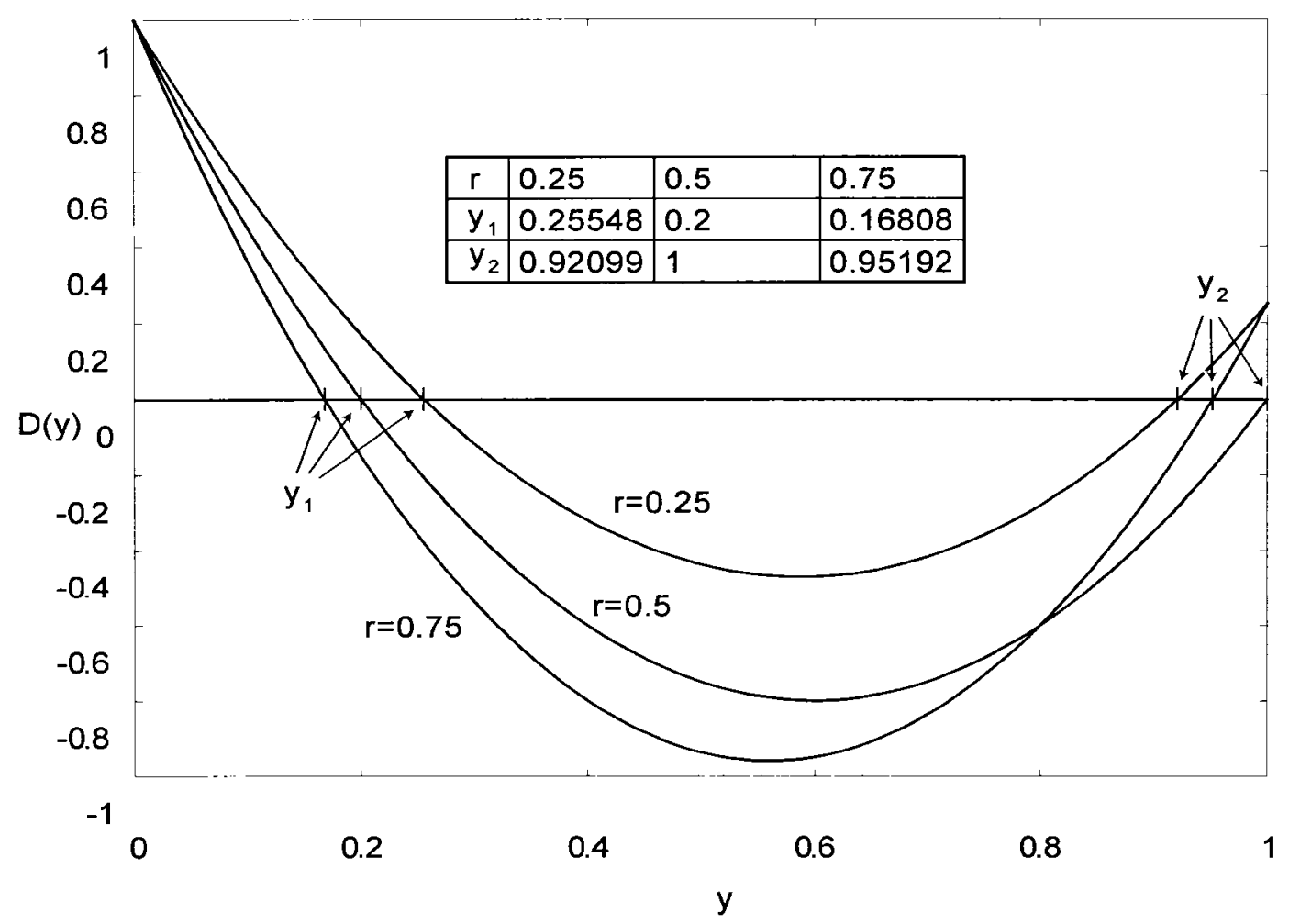

Figure 2.6: Coupled - JSQ: The Discriminant as a Function of Real $y$

\subsection{A Boundary Value Problem Solution for $P(x, 0)$}

The full analysis of this network requires the full specification of $P(x, y)$. For this, both $P(0, y)$ and $P(x, 0)$ are needed. In this section, $P(x, 0)$ is derived in a very similar manner to the way $P(0, y)$ is found in the previous section.

\subsubsection{Analysis of the Kernel}

When $h_{1}(x, y)$ is viewed as a polynomial in $x$, for every value of $y$ there are two values of $x$, say $x_{+}(y)$ and $x_{-}(y)$ such that $h_{1}\left(x_{+}(y), y\right)=h_{1}\left(x_{-}(y), y\right)=0$. These functions are presented above in (2.81). 
Lemma 2.4.1 The algebraic function $x(y)$ defined by $h_{1}(x(y), y)=0$, has two real branch points $0<y_{1}<y_{2} \leq 1$.

Proof: The branch points of $x(y)$ are the zeros of its determinant

$$
D_{2}(y)=4\left(r^{2}+1\right) y^{2}-4(r+1) y+1
$$

which are given by

$$
y_{1}=\frac{r+1-\sqrt{2 r}}{2\left(r^{2}+1\right)}, \quad y_{2}=\frac{r+1+\sqrt{2 r}}{2\left(r^{2}+1\right)}
$$

Note that $D_{2}(0)=1>0$ and $D_{2}(1)=(2 r-1)^{2} \geq 0$. Also for $y^{\prime}=\frac{1}{2(r+1)}$, $D\left(y^{\prime}\right)<0$. Therefore, it must be that $0<y_{1}<y^{\prime}<y_{2} \leq 1$. In fact, $y_{2}=1$ if and only if $r=1 / 2$ (i.e. $\lambda=\nu$ ).

Figure 2.6 shows the graph of $D_{2}(y)$ for real $y \in[0,1]$ and varying values of $r$ including $r=\frac{1}{2}$ which gives $y_{2}=1$.

Lemma 2.4.2 For $y \in\left[y_{1}, y_{2}\right], x(y)$ lies on the closed contour $R$, which is symmetric with the real line and defined by

$$
|x|^{2}=m(\operatorname{Re}(x))
$$

where

$$
m(\delta)=\frac{\zeta(\delta)^{2}}{2 r}
$$

and

$$
\zeta(\delta)=\frac{4 r \delta+1}{2(r+1)}
$$

It further holds that

$$
|x| \leq \beta
$$

where

$$
\beta=\frac{r+1+\sqrt{2 r}}{2\left(r^{2}+1\right) \sqrt{2 r}}
$$




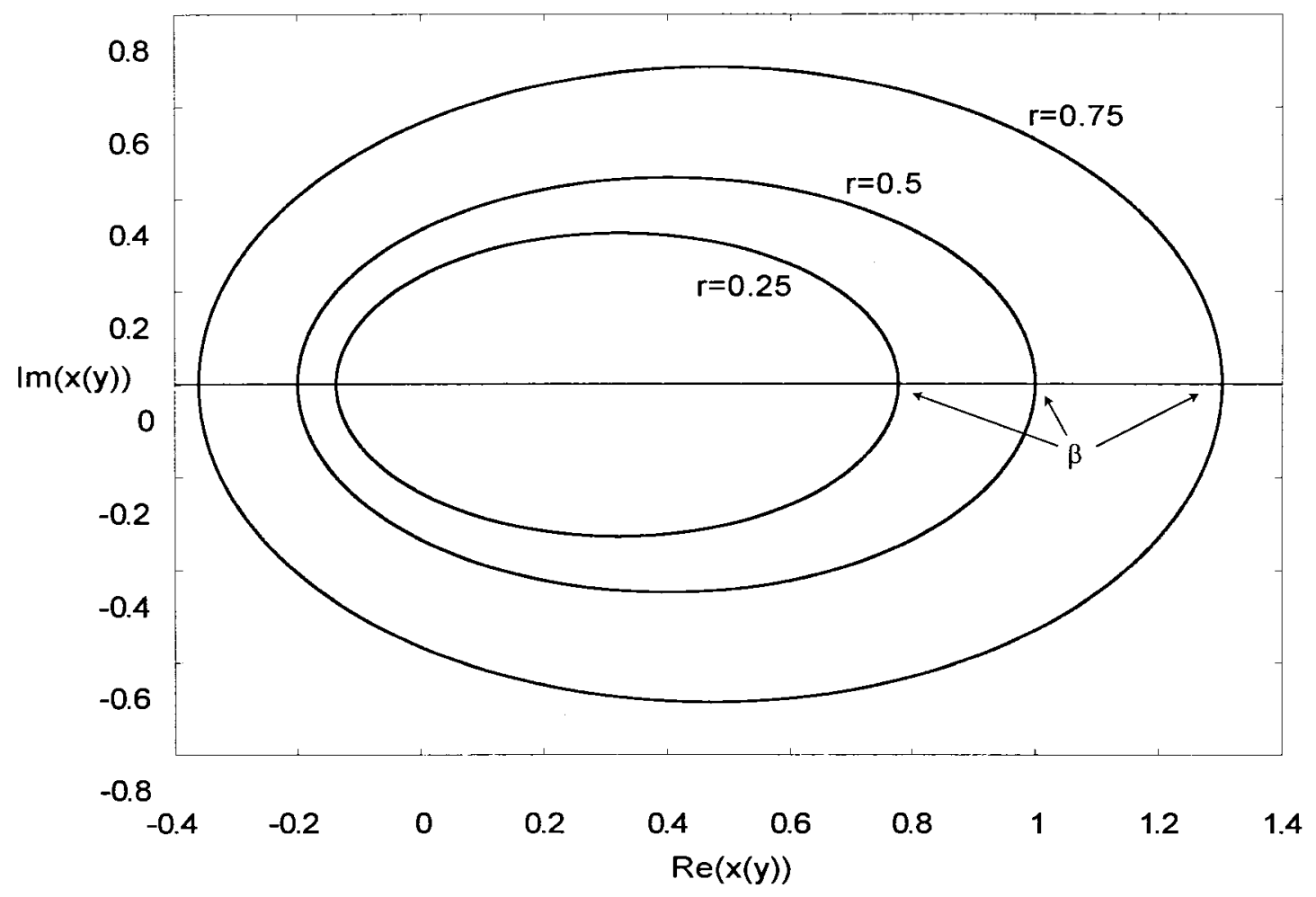

Figure 2.7: Coupled - JSQ: The Contour $R$ 
Proof: For all $y \in\left(y_{1}, y_{2}\right)$, we see $D_{2}(y)<0$, and therefore, $x(y)$ is complex. So,

$$
\begin{aligned}
\operatorname{Re}(x(y)) & =\frac{2(r+1) y-1}{4 r} \\
\operatorname{Im}(x(y)) & = \pm \frac{\sqrt{8 r y^{2}-[2(r+1) y-1]^{2}}}{4 r}
\end{aligned}
$$

and

$$
|x(y)|^{2}=\frac{y^{2}}{2 r}
$$

which means

$$
|x(y)|=\frac{y}{\sqrt{2 r}}
$$

As, $|x(y)|$ is clearly increasing with $y>0$, the maximum modulus of $x(y)$ for $y \in\left[y_{1}, y_{2}\right]$ is $\left|x\left(y_{2}\right)\right|$. Let $\beta$ be this value.

$\zeta(\delta)$ is derived by solving $(2.119)$ for $y$.

Figure 2.7 shows plots of the contour $R$ for different values of $r$. Note how the contour escapes the unit circle if $r>\frac{1}{2}$. The next corollary explains this behavior.

\section{Corollary 2.4.1}

$$
\begin{aligned}
& r=\frac{1}{2} \Leftrightarrow \beta=1 \\
& r<\frac{1}{2} \Leftrightarrow \beta>1 \\
& r>\frac{1}{2} \Leftrightarrow \beta<1
\end{aligned}
$$

Proof: When $\beta=1$ it must also be true that

$$
8 r^{5}+8 r^{3}-r^{2}-1=0
$$

which can be factored to

$$
2(r-1 / 2)\left(4 r^{4}+2 r^{3}+5 r^{2}+2 r+1\right)=0
$$

For $r>0,\left(4 r^{4}+2 r^{3}+5 r^{2}+2 r+1\right)>0$. Therefore, $\beta=1$, only for $r=1 / 2$. Simple calculations confirm

$$
\left.\beta\right|_{r=1 / 4} \approx 1.302,\left.\quad \beta\right|_{r=3 / 4} \approx 0.7772
$$


As $\beta$ is continuous in $r>0$ the proof is complete.

Corollary 2.4.2 Let $R^{+}$be the interior of $R$. For all values of $r>0$, it is always true that $0 \in R^{+}$.

Proof: Clearly, $\operatorname{Re}(x(y))$ is increasing in $y$. Therefore,

$$
\max \{\operatorname{Re}(x(y))\}=x\left(y_{2}\right)=\beta>0, \quad r>0
$$

It follows that

$$
\min \{\operatorname{Re}(x(y))\}=x\left(y_{1}\right)=\frac{2 r-(r+1) \sqrt{2 r}}{4 r\left(r^{2}+1\right)}
$$

It is readily confirmed that for $r>0,(2 r-(r+1) \sqrt{2 r}) \neq 0$ and, therefore, $x\left(y_{1}\right) \neq 0$. In fact, as $\left.x\left(y_{1}\right)\right|_{r=1 / 2}=-1 / 15$ and $x\left(y_{1}\right)$ is continuous for $r>0$, it can be concluded that $x\left(y_{1}\right)<0$ for all $r>0$. All values of $x$ in the interval $\left(x\left(y_{1}\right), x\left(y_{2}\right)\right)$ are in $R^{+}$. Therefore, as $x\left(y_{1}\right)<0<x\left(y_{2}\right)$ it must also be true that $0 \in R^{+}$.

\subsubsection{Analytic Continuation}

Lemma 2.4.3 The function $P(x, 0)$ is analytic in the domain $R^{+}$and satisfies for $x \in R$ the condition

$$
\operatorname{Im}[(2 r x+1) P(x, 0)]=\operatorname{Im}\left[\frac{2 x \eta(x)-x-\eta(x)^{2}}{x-\eta(x)^{2}}\right] P(0,0)
$$

where

$$
\eta(x)=|x| \sqrt{2 r}
$$

Proof: The proof of this lemma is similar to the proof for Lemma 2.3.3.

For zero pairs $(x, y)$ of the kernel $h_{1}(x, y)$ for which $P(x, y)$ is finite, from $(2.21)$

$$
\begin{aligned}
P(x, 0) & =-\frac{h_{3}(x, y)}{h_{2}(x, y)} P(0, y)-\frac{h_{4}(x, y)}{h_{2}(x, y)} P(0,0) \\
& =\frac{1}{2 r x+1} P(0, y)+\frac{2 x y-x-y^{2}}{(2 r x+1)\left(x-y^{2}\right)} P(0,0)
\end{aligned}
$$


If $\beta \leq 1$ then $P(x, 0)$ is clearly analytic in $R^{+}$as $x \in R^{+}$implies $|x|<1$ and for $y=y(x),|y|<1$.

If $\beta>1$ it remains to be seen if the right hand side of (2.130) is analytic for $x \in R^{+}$.

In Lemma 2.3.3 it is concluded that the only solution of concern to $x-y^{2}=0$ is $(x, y)=\left(\frac{1}{r^{2}}, \frac{1}{r}\right)$. However, $y=\frac{1}{r}$ is outside the unit circle and $x=\frac{1}{r^{2}}>\beta$. Consider the possibility of $2 r x+1=0$ for $x \in R \cup R^{+} . x=-\frac{1}{2 r}$ is real and negative for $r>0$. Equating $x\left(y_{1}\right)$, using (2.119), with $-\frac{1}{2 r}$ would require $y_{1}=-\frac{1}{2(r+1)}$. As $y_{1}>0$ this is clearly not possible for $r>0$. In fact, for $r=1 / 4, x\left(y_{1}\right) \approx-0.3613$ while $-\frac{1}{2 r}=-2$. Therefore, $x\left(y_{1}\right)>-\frac{1}{2 r}$ for $r>0$ and as $x\left(y_{1}\right)$ is the smallest point on $R$, it can be concluded that $(2 r x+1) \neq 0$ for $x \in R \cup R^{+}$.

So, the right hand side of (2.130) is analytic for $x \in R^{+}$. As, $P(x, 0)$ is analytic, certainly $(2 r x+1) P(x, 0)$ is also analytic for $x \in R^{+}$. On the boundary, $P(0, y(x))$ is strictly real. So, multiplying (2.130) through by $(2 r x+1)$ allows the derivation of $(2.128)$

The function $\eta(x)$ is derived simply by solving (2.122) for $y$. It allows the specification of the boundary condition using $x$ alone.

\subsubsection{The Boundary Value Problem}

The determination of $P(x, 0)$ reduces to the determination of the solution of the following Riemann-Hilbert boundary value problem on the contour $R$ : Determine a $P(x, 0)$ such that

1. $P(x, 0)$ is analytic for $x \in R^{+}$and continuous for $x \in R \cup R^{+}$

2. $\operatorname{Re}[i(2 r x+1) P(x, 0)]=d(x)$ for $x \in L$ where

$$
d(x)=\operatorname{Im}\left[\frac{2 x \eta(x)-x-\eta(x)^{2}}{x-\eta(x)^{2}}\right] P(0,0)
$$

The conformal mapping

$$
z=g(x): R^{+} \rightarrow C^{+}
$$

and its inverse

$$
x=g_{0}(z): C^{+} \rightarrow R^{+}
$$


can be introduced to reduce the Riemann-Hilbert problem on $R$ to a Dirichlet problem on the unit circle. The solution is given by

$$
P(x, 0)=-\frac{1}{2 \pi(2 r x+1)}\left[\oint_{C} d\left(g_{0}(w)\right) \frac{w+g(x)}{w-g(x)} \frac{\mathrm{d} w}{w}+\kappa_{2}\right], \quad x \in R \cup R^{+}
$$

where $\kappa_{2}$ is a constant. As $\pi_{0,0}$ is known from (2.28), $\kappa_{2}$ can easily be determined by evaluating (2.131) for $x=0$.

For $x \in R \cup R^{+}$the derivative of $P(x, 0)$ is given as

$$
\frac{\mathrm{d}}{\mathrm{d} x} P(x, 0)=-\frac{1}{2 r x+1}\left[2 r P(x, 0)+\frac{1}{\pi} \oint_{C} d\left(g_{0}(w)\right) \frac{g^{\prime}(x)}{(w-g(x))^{2}} \mathrm{~d} w\right], \quad x \in R \cup R^{+}
$$

The technique outlined in section 2.3.4 can be applied in an identical fashion to compute the conformal map $g$, its inverse $g_{0}$, and its derivative $g^{\prime}$.

Thus, $P(x, 0)$ for $x \in R \cup R^{+}$is found.

A limitation of this solution for $P(x, 0)$ is that for $r<\frac{1}{2}, 1 \notin R \cup R^{+}$. This means that it is not possible to directly compute $P(1,0)$ and $\left.\frac{\mathrm{d}}{\mathrm{d} x} P(x, 0)\right|_{x=1}$ using (2.131) and (2.132). This issue does arise frequently in solutions to boundary value problems. The standard way of resolving this issue is to compute a Taylor series expansion of $P(x, 0)$ around some point that is in $R \cup R^{+}$and then evaluate this approximation at $x=1$. However, to compute a Taylor series approximation would require the ability to take an $n^{\text {th }}$ order derivative of $P(x, 0)$ and hence the conformal map $g$. This seems only possible with explicit forms for $g$.

\subsection{Conclusion}

The long-run distribution of the queue lengths of this two-node network is now fully defined. The characteristic function $P(x, y)$ can be expressed as a combination of $P(0, y)$ and $P(x, 0)$ through the functional equation (1.3). Both $P(0, y)$ and $P(x, 0)$ are determined as the solution to complex boundary value problems. 
The derivatives of $P(0, y)$ and $P(x, 0)$ can be used to compute the mean queue lengths. In fact, because of the simplistic service coupling used, the mean queue lengths are computed without even solving the boundary value problems.

Further research should be made into loosening the restrictions we placed on the coupled service rate. The following issues would arise

1. It may not be possible to find a $\gamma(y)$ such that $h_{2}(\gamma(y), y)=h_{3}(\gamma(y), y)=$ 0 . This may prevent the explicit determination of $\pi_{0,0}$

2. The quotient $\frac{h_{2}(x, y)}{h_{3}(x, y)}$ may have poles and zeros hindering the analytic continuation of $P(0, y)$ and $P(x, 0)$ along their respective contours. And,

3. If the quotient $\frac{h_{2}(x, y)}{h_{3}(x, y)}$ is not strictly real it may be difficult to determine a boundary condition that does not express $P(0, y)$ as a function of $P(x, 0)$ and vice-versa.

Resolving the last two items may require expanding upon the technique used in specifying the boundary condition for $P(x, 0)$ in $(2.128)$ where $P(x, 0)$ is multiplied by an analytic function of $x$ to allow the specification of the boundary condition for $P(x, 0)$ without including $P(0, y)$.

It would also be nice to consider an asymmetric JSQ model. In this model, rather than having the same service rate for each queue, each queue would have its own individual rate. To accommodate this change, specifying $Y_{2}(t)$ as the absolute difference in the two queue lengths would no longer suffice. If instead $Y_{2}(t)$ is defined as the non-absolute difference (i.e. $X_{1}(t)-X_{2}(t)$ ), $Y_{2}(t)$ could range between positive and negative values. In this case, the process $Y(t)=\left(Y_{1}(t), Y_{2}(t)\right)$ would cease to be a random walk in the top-right quarter plane; it would encompass the entire right half plane. This may add interesting elements to the analysis.

The boundary value technique used in this thesis is only applicable to networks of two queues. However, the success of the ad-hoc methods used in the "Preliminary Results" section indicates they could perhaps be applied to a JSQ network with more than two queues. Further research along these lines may yield some very significant results. 


\section{Chapter 3}

\section{Jackson Network with Coupled Processors}

In this chapter another two-queue network with coupled processors is considered. This network is far more complicated than the coupled JSQ model analyzed in the previous chapter. In spite of this complexity the characteristic function of the joint queue length distribution is fully defined as the solution to a complex boundary value problem. Additionally, forms for the average queue length are presented.

\subsection{Model Description}

The Jackson network is a standard model well analyzed in literature. Arrivals at each queue $(i=1,2)$ are a Poisson process with rate $\lambda_{i}$. Service times for each queue are exponentially distributed with rate $\nu_{i}$. After service is completed, a customer will leave the network with probability $p_{i}$, or, remain in the network by entering the other queue and await service with probability $1-p_{i}$. Figure 3.1 illustrates how customers can flow through a Jackson network.

There are many "real-world" applications to this network. For example the two servers may represent operators in a call-center. Each operator may receive outside calls independently. There is a certain probability, however, that a single operator will not be able to fully serve a customer, perhaps because of a limited skill set, in which case a customer would be transferred to another operator to complete the service. 


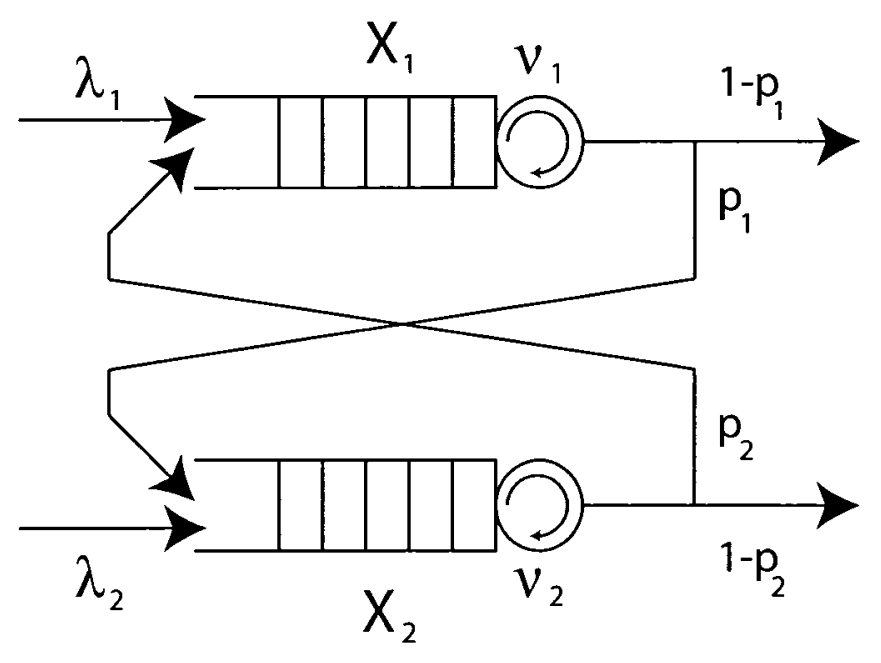

Figure 3.1: Coupled Jackson: Model Description

In this document a variation to this standard model is considered. Namely, coupling of the two servers is introduced. When both queues are non-empty the service rates are $\nu_{1}$ and $\nu_{2}$; however, when one of the queues is empty the service rate of the other increases. If the first queue is empty then the service rate of the second increases from $\nu_{2}$ to $\nu_{2}^{*}$. Likewise, the service rate of the first will increase from $\nu_{1}$ to $\nu_{1}^{*}$ when the second is empty.

In the call-center application, server coupling could be likened to an idle operator assisting a busy operator in some manner. The busy operator will be able to serve customers faster with the idle operator assisting.

Some simpler two node networks are variations of the more generic Jackson network. For example, if $p_{1}=p_{2}=0$ we have the parallel coupled queues analyzed by Blanc [1]. Also, if $\lambda_{2}=0, p_{1}=1$ and $p_{2}=0$ we have the tandem coupled queues described by Resing [13]. The results of this thesis generalize the results from these simpler networks.

It should be noted that van Leeuwaarden also considers this model in chapter 10 of his Ph.D. thesis [11]. Unfortunately, I had completed my own analysis of the problem before realizing that it had already been addressed. This was careless especially as I had spent so much time reading chapter 9 of his thesis and apparently overlooking chapter 10. Anyways, my analysis is presented. 
It corresponds well with van Leeuwaarden's. In fact, I am a bit more thorough in considering all the possible variations of the model.

\subsubsection{The Balance Equations}

Let $X_{i}(t)$ be the depth of queue $i, i=1,2$, at time $t$. Therefore, $X(t)=$ $\left(X_{1}(t), X_{2}(t)\right)$ is a Markov process that is irreducible and aperiodic. Let $\pi_{m, n}=\lim _{t \rightarrow \infty} \mathbb{P}\left[X_{1}(t)=m, X_{2}(t)=n\right]$. If $X(t)$ is ergodic then $\pi$ is the unique stationary distribution. See [6] for a discussion on the conditions for which this coupled Jackson network is ergodic.

Figure 3.2 illustrates the long-run state transition rates for $X(t)$. Notice how the coupled service rates $\left(\nu_{i}^{*}, i=1,2\right)$ apply when either $X_{1}=0$ or $X_{2}=0$.

In Figure 3.2, the shaded regions group states that have the same pattern of ingress and egress rates. Each shaded region has a corresponding balance equation. The stationary distribution $\pi=\left\{\pi_{m, n}: m, n=0,1,2,3, \ldots\right\}$ is the unique solution to this system of equations. 


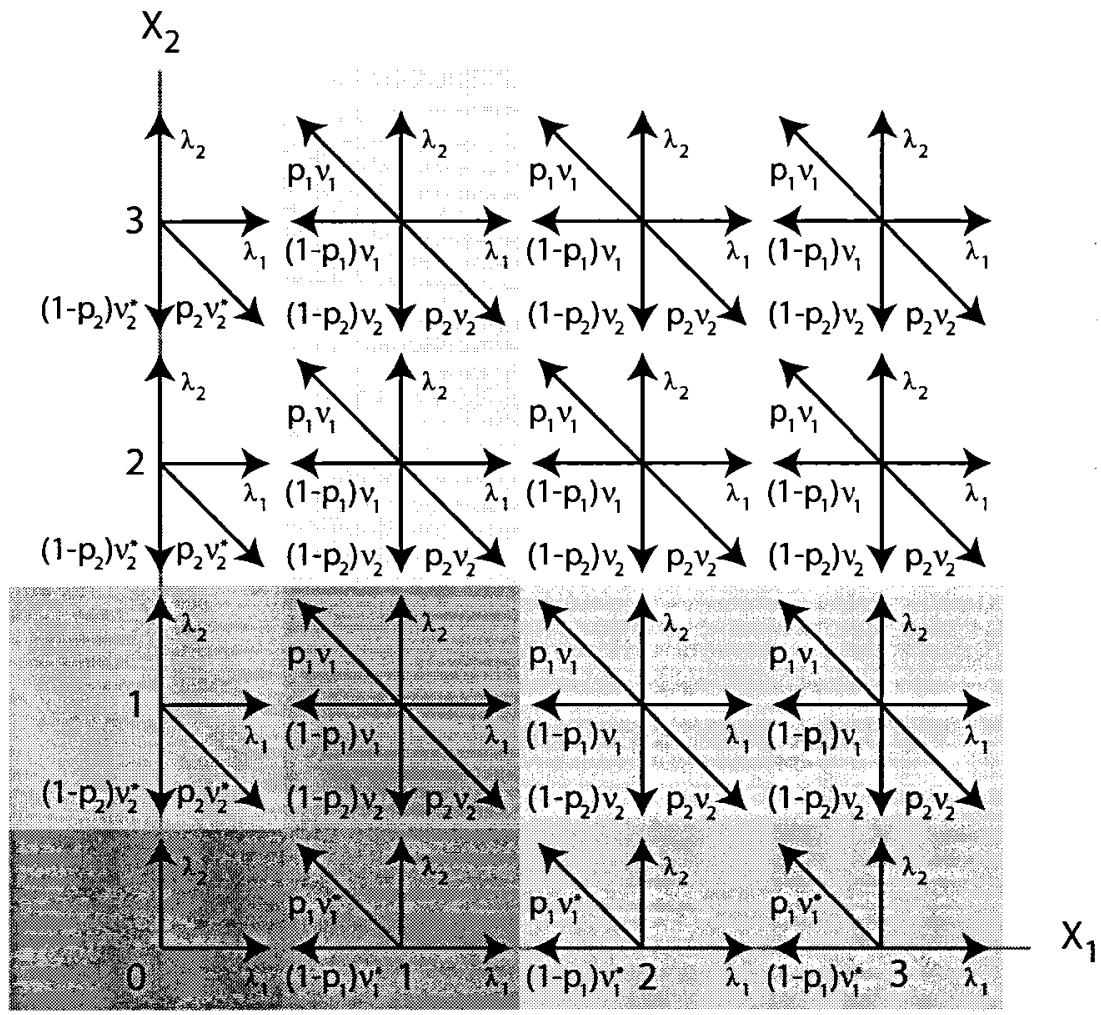

Figure 3.2: Coupled Jackson: State Transition Rates 
The balance equations are, for $m, n=2,3,4, \ldots$

$$
\begin{aligned}
\left(\lambda_{1}+\lambda_{2}\right) \pi_{0,0}= & \left(1-p_{1}\right) \nu_{1}^{*} \pi_{1,0}+\left(1-p_{2}\right) \nu_{2}^{*} \pi_{0,1} \\
\left(\lambda_{1}+\lambda_{2}+\nu_{1}^{*}\right) \pi_{1,0}= & \lambda_{1} \pi_{0,0}+\left(1-p_{1}\right) \nu_{1}^{*} \pi_{2,0} \\
& +p_{2} \nu_{2}^{*} \pi_{0,1}+\left(1-p_{2}\right) \nu_{2} \pi_{1,1} \\
\left(\lambda_{1}+\lambda_{2}+\nu_{1}^{*}\right) \pi_{m, 0}= & \lambda_{1} \pi_{m-1,0}+\left(1-p_{1}\right) \nu_{1}^{*} \pi_{m+1,0} \\
& +p_{2} \nu_{2} \pi_{m-1,1}+\left(1-p_{2}\right) \nu_{2} \pi_{m, 1} \\
\left(\lambda_{1}+\lambda_{2}+\nu_{2}^{*}\right) \pi_{0,1}= & \lambda_{2} \pi_{0,0}+p_{1} \nu_{1}^{*} \pi_{1,0} \\
& +\left(1-p_{1}\right) \nu_{1} \pi_{1,1}+\left(1-p_{2}\right) \nu_{2}^{*} \pi_{0,2} \\
\left(\lambda_{1}+\lambda_{2}+\nu_{2}^{*}\right) \pi_{0, n}= & \lambda_{2} \pi_{0, n-1}+p_{1} \nu_{1} \pi_{1, n-1} \\
& +\left(1-p_{1}\right) \nu_{1} \pi_{1, n}+\left(1-p_{2}\right) \nu_{2}^{*} \pi_{0, n+1} \\
\left(\lambda_{1}+\lambda_{2}+\nu_{1}+\nu_{2}\right) \pi_{1,1}= & \lambda_{1} \pi_{0,1}+\lambda_{2} \pi_{1,0} \\
& +p_{1} \nu_{1}^{*} \pi_{2,0}+\left(1-p_{1}\right) \nu_{1} \pi_{2,1} \\
& +p_{2} \nu_{2}^{*} \pi_{0,2}+\left(1-p_{2}\right) \nu_{2} \pi_{1,2} \\
\left(\lambda_{1}+\lambda_{2}+\nu_{1}+\nu_{2}\right) \pi_{m, 1}= & \lambda_{1} \pi_{m-1,1}+\lambda_{2} \pi_{m, 0} \\
& +p_{1} \nu_{1}^{*} \pi_{m+1,0}+\left(1-p_{1}\right) \nu_{1} \pi_{m+1,1} \\
& +p_{2} \nu_{2} \pi_{m-1,2}+\left(1-p_{2}\right) \nu_{2} \pi_{m, 2} \\
\left(\lambda_{1}+\lambda_{2}+\nu_{1}+\nu_{2}\right) \pi_{1, n}= & \lambda_{1} \pi_{0, n}+\lambda_{2} \pi_{1, n-1} \\
& +p_{1} \nu_{1} \pi_{2, n-1}+\left(1-p_{1}\right) \nu_{1} \pi_{2, n} \\
& +p_{2} \nu_{2}^{*} \pi_{0, n+1}+\left(1-p_{2}\right) \nu_{2} \pi_{1, n+1} \\
\left(\lambda_{1}+\lambda_{2}+\nu_{1}+\nu_{2}\right) \pi_{m, n}= & \lambda_{1} \pi_{m-1, n}+\lambda_{2} \pi_{m, n-1} \\
& +p_{1} \nu_{1} \pi_{m+1, n-1}+\left(1-p_{1}\right) \nu_{1} \pi_{m+1, n} \\
& +p_{2} \nu_{2} \pi_{m-1, n+1}+\left(1-p_{2}\right) \nu_{2} \pi_{m, n+1} \\
&
\end{aligned}
$$

\subsubsection{The Functional Equation}

These balance equations can be combined to assemble the functional equation

(1.3) for this model. 
Multiplying (3.9) by $x^{m+1} y^{n+1}$ and then summing it for all valid states gives

$$
\begin{aligned}
& \left(\lambda_{1}+\lambda_{2}+\nu_{1}+\nu_{2}\right) \sum_{m=2}^{\infty} \sum_{n=2}^{\infty} x^{m+1} y^{n+1} \pi_{m, n}= \\
& \lambda_{1} \sum_{m=2}^{\infty} \sum_{n=2}^{\infty} x^{m+1} y^{n+1} \pi_{m-1, n}+\lambda_{2} \sum_{m=2}^{\infty} \sum_{n=2}^{\infty} x^{m+1} y^{n+1} \pi_{m, n-1}+ \\
& p_{1} \nu_{1} \sum_{m=2}^{\infty} \sum_{n=2}^{\infty} x^{m+1} y^{n+1} \pi_{m+1, n-1}+\left(1-p_{1}\right) \nu_{1} \sum_{m=2}^{\infty} \sum_{n=2}^{\infty} x^{m+1} y^{n+1} \pi_{m+1, n}+ \\
& p_{2} \nu_{2} \sum_{m=2}^{\infty} \sum_{n=2}^{\infty} x^{m+1} y^{n+1} \pi_{m-1, n+1}+\left(1-p_{2}\right) \nu_{2} \sum_{m=2}^{\infty} \sum_{n=2}^{\infty} x^{m+1} y^{n+1} \pi_{m, n+1}
\end{aligned}
$$

or,

$$
\begin{aligned}
& \left(\lambda_{1}+\lambda_{2}+\nu_{1}+\nu_{2}\right) x y \sum_{m=2}^{\infty} \sum_{n=2}^{\infty} x^{m} y^{n} \pi_{m, n}= \\
& \lambda_{1} x^{2} y \sum_{m=1}^{\infty} \sum_{n=2}^{\infty} x^{m} y^{n} \pi_{m, n}+\lambda_{2} x y^{2} \sum_{m=2}^{\infty} \sum_{n=1}^{\infty} x^{m} y^{n} \pi_{m, n}+ \\
& p_{1} \nu_{1} y^{2} \sum_{m=3}^{\infty} \sum_{n=1}^{\infty} x^{m} y^{n} \pi_{m, n}+\left(1-p_{1}\right) \nu_{1} y \sum_{m=3}^{\infty} \sum_{n=2}^{\infty} x^{m} y^{n} \pi_{m, n}+ \\
& p_{2} \nu_{2} x^{2} \sum_{m=1}^{\infty} \sum_{n=3}^{\infty} x^{m} y^{n} \pi_{m, n}+\left(1-p_{2}\right) \nu_{2} x \sum_{m=2}^{\infty} \sum_{n=3}^{\infty} x^{m} y^{n} \pi_{m, n}
\end{aligned}
$$


Similarly, multiplying (3.8) by $x y^{n+1},(3.7)$ by $x^{m+1} y$, and (3.6) by $x y$, summing through all valid states, and then combining with (3.10) gives

$$
\begin{aligned}
& \left(\lambda_{1}+\lambda_{2}+\nu_{1}+\nu_{2}\right) x y \sum_{m=1}^{\infty} \sum_{n=1}^{\infty} x^{m} y^{n} \pi_{m, n}= \\
& \lambda_{1} x^{2} y \sum_{m=0}^{\infty} \sum_{n=1}^{\infty} x^{m} y^{n} \pi_{m, n}+\lambda_{2} x y^{2} \sum_{m=1}^{\infty} \sum_{n=0}^{\infty} x^{m} y^{n} \pi_{m, n}+ \\
& p_{1} \nu_{1} y^{2} \sum_{m=2}^{\infty} \sum_{n=1}^{\infty} x^{m} y^{n} \pi_{m, n}+p_{1} \nu_{1}^{*} y^{2} \sum_{m=2}^{\infty} x^{m} \pi_{m, 0}+ \\
& \left(1-p_{1}\right) \nu_{1} y \sum_{m=2}^{\infty} \sum_{n=1}^{\infty} x^{m} y^{n} \pi_{m, n}+ \\
& p_{2} \nu_{2} x^{2} \sum_{m=1}^{\infty} \sum_{n=2}^{\infty} x^{m} y^{n} \pi_{m, n}+p_{2} v_{2}^{*} x^{2} \sum_{n=2}^{\infty} y^{n} \pi_{0, n}+ \\
& \left(1-p_{2}\right) \nu_{2} x \sum_{m=1}^{\infty} \sum_{n=2}^{\infty} x^{m} y^{n} \pi_{m, n}
\end{aligned}
$$

or,

$$
\begin{aligned}
& \left(\lambda_{1}+\lambda_{2}+\nu_{1}+\nu_{2}\right) x y\left[P(x, y)-P(x, 0)-P(0, y)+\pi_{0,0}\right]= \\
& \quad \lambda_{1} x^{2} y[P(x, y)-P(x, 0)]+\lambda_{2} x y^{2}[P(x, y)-P(0, y)]+ \\
& \quad\left[p_{1} \nu_{1} y^{2}+\left(1-p_{1}\right) \nu_{1} y\right]\left[P(x, y)-P(x, 0)-P(0, y)+\pi_{0,0}-\sum_{n=1}^{\infty} x y^{n} \pi_{1, n}\right]+ \\
& p_{1} \nu_{1}^{*} y^{2}\left[P(x, 0)-x \pi_{1,0}-\pi_{0,0}\right]+ \\
& \quad\left[p_{2} \nu_{2} x^{2}+\left(1-p_{2}\right) \nu_{2} x\right]\left[P(x, y)-P(x, 0)-P(0, y)+\pi_{0,0}-\sum_{m=1}^{\infty} x^{m} y \pi_{m, 1}\right]+ \\
& p_{2} v_{2}^{*} x^{2}\left[P(0, y)-y \pi_{0,1}-\pi_{0,0}\right]
\end{aligned}
$$

$\sum_{m=1}^{\infty} x^{m} y \pi_{m, 1}$ can be expressed as a combination of $P(x, 0), \pi_{0,1}$ and $\pi_{0,0}$ by summing (3.3) and (3.2). Likewise, $\sum_{n=1}^{\infty} x y^{n} \pi_{1, n}$ can be reduced to a combination of $P(0, y), \pi_{1,0}$ and $\pi_{0,0}$ by summing (3.5) and (3.4). Finally, $\pi_{0,1}$ and $\pi_{1,0}$ are eliminated using (3.1). The functional equation for $P(x, y)$ is, therefore,

$$
h_{1}^{*}(x, y) P(x, y)=h_{2}^{*}(x, y) P(x, 0)+h_{3}^{*}(x, y) P(0, y)+h_{4}^{*}(x, y) P(0,0)
$$


where,

$$
\begin{aligned}
h_{1}^{*}(x, y)= & \left(\lambda_{1}+\lambda_{2}+\nu_{1}+\nu_{2}\right) x y-\lambda_{1} x^{2} y-\lambda_{2} x y^{2}-p_{1} \nu_{1} y^{2}-\left(1-p_{1}\right) \nu_{1} y \\
& -p_{2} \nu_{2} x^{2}-\left(1-p_{2}\right) \nu_{2} x \\
h_{2}^{*}(x, y)= & \left(\nu_{1}-\nu_{1}^{*}+\nu_{2}\right) x y-p_{1}\left(\nu_{1}-\nu_{1}^{*}\right) y^{2}-\left(1-p_{1}\right)\left(\nu_{1}-\nu_{1}^{*}\right) y \\
& -p_{2} \nu_{2} x^{2}-\left(1-p_{2}\right) \nu_{2} x \\
h_{3}^{*}(x, y)= & \left(\nu_{1}+\nu_{2}-\nu_{2}^{*}\right) x y-p_{1} \nu_{1} y^{2}-\left(1-p_{1}\right) \nu_{1} y \\
& -p_{2}\left(\nu_{2}-\nu_{2}^{*}\right) x^{2}-\left(1-p_{2}\right)\left(\nu_{2}-\nu_{2}^{*}\right) x \\
h_{4}^{*}(x, y)= & -\left(\left(\nu_{1}-\nu_{1}^{*}\right)+\left(\nu_{2}-\nu_{2}^{*}\right)\right) x y+p_{1}\left(\nu_{1}-\nu_{1}^{*}\right) y^{2}+\left(1-p_{1}\right)\left(\nu_{1}-\nu_{1}^{*}\right) y \\
& +p_{2}\left(\nu_{2}-\nu_{2}^{*}\right) x^{2}+\left(1-p_{2}\right)\left(\nu_{2}-\nu_{2}^{*}\right) x
\end{aligned}
$$

To simplify the analysis of this network, a restriction is imposed on how the servicing of the two queues is coupled. When both queues are busy, their service rates are $p^{*} \nu_{1}$ and $\left(1-p^{*}\right) \nu_{2}$ respectively, where $0 \leq p^{*} \leq 1$. When the second queue is idle, the service rate of the first increases to $\nu_{1}$. Likewise, the service rate of the second queue increases to $\nu_{2}$ when the first queue is empty.

This form of coupling can be viewed as sharing a service resource between the two queues such that when both are operational, $p^{*}$ of one unit of service potential is allocated to the first queue and the remaining $1-p^{*}$ of the service unit is allocated to the second queue. When one of the queues is empty the fully service potential is allocated to the other. For example, say two machines are powered by the same $1000 \mathrm{~kW}$ generator. Each machine will operate at its full capacity only if it is drawing the entire $1000 \mathrm{~kW}$. However, when both machines are operating, $750 \mathrm{~kW}$ is allocated to one and $250 \mathrm{~kW}$ to the other. We assume that the first will only function at $75 \%$ of its full potential and the second at $25 \%$. Only when one machine is idle will the other operate at $100 \%$ because only then is the full $1000 \mathrm{~kW}$ of power available.

Under this restriction, the functional equation becomes:

$$
h_{1}(x, y) P(x, y)=h_{2}(x, y) P(x, 0)+h_{3}(x, y) P(0, y)+h_{4}(x, y) P(0,0)
$$


where

$$
\begin{aligned}
h_{1}(x, y)= & \left(\lambda_{1}+\lambda_{2}+p^{*} \nu_{1}+\left(1-p^{*}\right) \nu_{2}\right) x y-\lambda_{1} x^{2} y-\lambda_{2} x y^{2} \\
& -p^{*} p_{1} \nu_{1} y^{2}-p^{*}\left(1-p_{1}\right) \nu_{1} y-\left(1-p^{*}\right) p_{2} \nu_{2} x^{2}-\left(1-p^{*}\right)\left(1-p_{2}\right) \nu_{2} x \\
h_{2}(x, y)= & -\left(1-p^{*}\right)\left[\left(\nu_{1}-\nu_{2}\right) x y-p_{1} \nu_{1} y^{2}-\left(1-p_{1}\right) \nu_{1} y+p_{2} \nu_{2} x^{2}+\left(1-p_{2}\right) \nu_{2} x\right] \\
h_{3}(x, y)= & p^{*}\left[\left(\nu_{1}-\nu_{2}\right) x y-p_{1} \nu_{1} y^{2}-\left(1-p_{1}\right) \nu_{1} y+p_{2} \nu_{2} x^{2}+\left(1-p_{2}\right) \nu_{2} x\right] \\
h_{4}(x, y)= & \left(\left(1-p^{*}\right) \nu_{1}+p^{*} \nu_{2}\right) x y-\left(1-p^{*}\right) p_{1} \nu_{1} y^{2}-\left(1-p^{*}\right)\left(1-p_{1}\right) \nu_{1} y \\
& -p^{*} p_{2} \nu_{2} x^{2}-p^{*}\left(1-p_{2}\right) \nu_{2} x
\end{aligned}
$$

The service coupling has been restricted in this manner so that $h_{2}(x, y) / h_{3}(x, y)$ is always real. This property simplifies the specification of a boundary condition (3.79).

Some other restrictions are placed on parameters to ensure we are analyzing a reasonable network. These restrictions are:

1. We must have $\nu_{1}>0$ and $\nu_{2}>0$

2. At least one of $\lambda_{1}$ and $\lambda_{2}$ must be non-zero

3. If $p_{1}=0$ then $\lambda_{2}>0$

4. If $p_{2}=0$ then $\lambda_{1}>0$

5. Both $p_{1}$ and $p_{2}$ cannot be 1 .

In the development that follows, all permissable configurations of this coupled Jackson network are considered.

\subsection{Some Preliminary Results}

Directly from the functional equation (3.13) some significant results can be derived. These results are only available because of the restricted coupling. The first two results depend on finding a function $\gamma(y)$ such that $h_{2}(\gamma(y), y)=$ $h_{3}(\gamma(y), y)=0$. With the simplified coupling it is seen that

$$
\gamma(y)= \begin{cases}\frac{-\left[\left(\nu_{1}-\nu_{2}\right) y+\left(1-p_{2}\right) \nu_{2}\right]+\sqrt{\left[\left(\nu_{1}-\nu_{2}\right) y+\left(1-p_{2}\right) \nu_{2}\right]^{2}+4 p_{2} \nu_{1} \nu_{2}\left(p_{1} y+1-p_{1}\right) y}}{2 p_{2} \nu_{2}} & p_{2}>0 \\ \frac{\nu_{1}\left(p_{1} y+1-p_{1}\right) y}{\nu_{2}+\left(\nu_{1}-\nu_{2}\right) y} & p_{2}=0\end{cases}
$$

However, no such function can be found for a more general form of coupling. 


\subsection{1 $\pi_{0,0}$}

In this section $\pi_{0,0}$ is computed which is the long-run probability that both queues are empty.

With $x=\gamma(y)$ such that $h_{2}(\gamma(y), y)=h_{3}(\gamma(y), y)=0$, the functional equation (3.13) reduces to

$$
P(\gamma(y), y)=\frac{h_{4}(\gamma(y), y)}{h_{1}(\gamma(y), y)} P(0,0)
$$

It is possible to rewrite $h_{1}(x, y)$ and $h_{4}(x, y)$ as

$$
\begin{aligned}
h_{1}(x, y)= & h_{3}(x, y)+x\left[\left(\lambda_{1}+\lambda_{2}+\nu_{2}\right) y-\lambda_{1} x y-\lambda_{2} y^{2}\right. \\
& \left.-p_{2} \nu_{2} x-\left(1-p_{2}\right) \nu_{2}\right] \\
h_{4}(x, y)= & -h_{3}(x, y)+\nu_{1} y\left[x-p_{1} y-\left(1-p_{1}\right)\right]
\end{aligned}
$$

It follows that

$$
\begin{aligned}
h_{1}(\gamma(y), y)= & \gamma(y)\left[\left(\lambda_{1}+\lambda_{2}+\nu_{2}\right) y-\lambda_{1} \gamma(y) y-\lambda_{2} y^{2}\right. \\
& \left.-p_{2} \nu_{2} \gamma(y)-\left(1-p_{2}\right) \nu_{2}\right] \\
h_{4}(\gamma(y), y)= & \nu_{1} y\left[\gamma(y)-p_{1} y-\left(1-p_{1}\right)\right]
\end{aligned}
$$

As, $\gamma(1)=1$ and $P(1,1)=1$

$$
\pi_{0,0}=\lim _{y \rightarrow 1} \frac{h_{1}(\gamma(y), y)}{h_{4}(\gamma(y), y)}
$$

However, $h_{1}(\gamma(1), 1)=h_{4}(\gamma(1), 1)=0$ so l'Hôpital's rule must be applied to evaluate the limit. First,

$$
\begin{aligned}
h_{1}(\gamma(y), y)= & \gamma(y)\left[\left(\lambda_{1}+\lambda_{2}+\nu_{2}\right) y-\lambda_{1} \gamma(y) y\right. \\
& \left.-\lambda_{2} y^{2}-p_{2} \nu_{2} \gamma(y)-\left(1-p_{2}\right) \nu_{2}\right] \\
\frac{\mathrm{d}}{\mathrm{d} y} h_{1}(\gamma(y), y)= & \gamma^{\prime}(y)\left[\left(\lambda_{1}+\lambda_{2}+\nu_{2}\right) y-\lambda_{1} \gamma(y) y\right. \\
& \left.-\lambda_{2} y^{2}-p_{2} \nu_{2} \gamma(y)-\left(1-p_{2}\right) \nu_{2}\right] \\
& +\gamma(y)\left[\lambda_{1}+\lambda_{2}+\nu_{2}-\lambda_{1} \gamma^{\prime}(y) y-\lambda_{1} \gamma(y)\right. \\
& \left.-2 \lambda_{2} y-p_{2} \nu_{2} \gamma^{\prime}(y)\right] \\
\left.\frac{\mathrm{d}}{\mathrm{d} y} h_{1}(\gamma(y), y)\right|_{y=1}= & -\lambda_{2}+\nu_{2}-\lambda_{1} \gamma^{\prime}(1)-p_{2} \nu_{2} \gamma^{\prime}(1)
\end{aligned}
$$


and

$$
\begin{aligned}
h_{4}(\gamma(y), y)= & \nu_{1} \gamma(y)\left[\gamma(y)-p_{1} y-\left(1-p_{1}\right)\right] \\
\frac{\mathrm{d}}{\mathrm{d} y} h_{4}(\gamma(y), y)= & \nu_{1} \gamma^{\prime}(y)\left[\gamma(y)-p_{1} y-\left(1-p_{1}\right)\right] \\
& +\nu_{1} \gamma(y)\left[\gamma^{\prime}(y)-p_{1}\right] \\
\left.\frac{\mathrm{d}}{\mathrm{d} y} h_{4}(\gamma(y), y)\right|_{y=1}= & \nu_{1}\left[\gamma^{\prime}(1)-p_{1}\right]
\end{aligned}
$$

Also, for $p_{2} \neq 0$,

$$
\begin{aligned}
\gamma^{\prime}(y)= & \frac{-\left(\nu_{1}-\nu_{2}\right)}{2 p_{2} \nu_{2}} \\
& +\frac{\left[\left(\nu_{1}-\nu_{2}\right) y+\left(1-p_{2}\right) \nu_{2}\right]\left(\nu_{1}-\nu_{2}\right)+4 p_{1} p_{2} \nu_{1} \nu_{2} y+2\left(1-p_{1}\right) p_{2} \nu_{1} \nu_{2}}{2 p_{2} \nu_{2} \sqrt{\left[\left(\nu_{1}-\nu_{2}\right) y+\left(1-p_{2}\right) \nu_{2}\right]^{2}+4 p_{2} \nu_{1} \nu_{2}\left(p_{1} y+1-p_{1}\right) y}} \\
\gamma^{\prime}(1)= & \frac{p_{1} \nu_{1}+\nu_{2}}{\nu_{1}+p_{2} \nu_{2}}
\end{aligned}
$$

Likewise; for $p_{2}=0$,

$$
\begin{aligned}
\gamma^{\prime}(y)= & \nu_{1} \frac{\left[\nu_{2}+\left(\nu_{1}-\nu_{2}\right) y\right]\left(2 p_{1} y+1-p_{1}\right)}{\left[\nu_{2}+\left(\nu_{1}-\nu_{2}\right) y\right]^{2}} \\
& -\nu_{1} \frac{\left(\nu_{1}-\nu_{2}\right)\left[p_{1} y^{2}+\left(1-p_{1}\right) y\right]}{\left[\nu_{2}+\left(\nu_{1}-\nu_{2}\right) y\right]^{2}} \\
\gamma^{\prime}(1)= & \frac{p_{1} \nu_{1}+\nu_{2}}{\nu_{1}}
\end{aligned}
$$


The limit is then evaluated as

$$
\begin{aligned}
\pi_{0,0}= & \lim _{y \rightarrow 1} \frac{\frac{\mathrm{d}}{\mathrm{d} y} h_{1}(\gamma(y), y)}{\frac{\mathrm{d}}{\mathrm{d} y} h_{4}(\gamma(y), y)} \\
= & \frac{\left.\frac{\mathrm{d}}{\mathrm{d} y} h_{1}(\gamma(y), y)\right|_{y=1}}{\left.\frac{\mathrm{d}}{\mathrm{d} y} h_{4}(\gamma(y), y)\right|_{y=1}} \\
= & \frac{-\lambda_{2}+\nu_{2}-\lambda_{1} \gamma^{\prime}(1)-p_{2} \nu_{2} \gamma^{\prime}(1)}{\nu_{1}\left[\gamma^{\prime}(1)-p_{1}\right]} \\
= & \frac{-\lambda_{2}+\nu_{2}-\lambda_{1}\left(\frac{p_{1} \nu_{1}+\nu_{2}}{\nu_{1}+p_{2} \nu_{2}}\right)-p_{2} \nu_{2}\left(\frac{p_{1} \nu_{1}+\nu_{2}}{\nu_{1}+p_{2} \nu_{2}}\right)}{\nu_{1}\left[\left(\frac{p_{1} \nu_{1}+\nu_{2}}{\nu_{1}+p_{2} \nu_{2}}\right)-p_{1}\right]} \\
= & 1-\frac{\lambda_{1}\left(p_{1} \nu_{1}+\nu_{2}\right)}{\left(1-p_{1} p_{2}\right) \nu_{1} \nu_{2}}-\frac{\lambda_{2}\left(\nu_{1}+p_{2} \nu_{2}\right)}{\left(1-p_{1} p_{2}\right) \nu_{1} \nu_{2}}
\end{aligned}
$$

This result holds for $p_{2} \geq 0$. In fact, (3.32) gives the long-run probability that both queues are empty, for all meaningful configurations of the coupled Jackson network.

An alternative, and somewhat simpler technique of analysis, uses the traffic equations for the Jackson network. This technique gives the same result as we shall show.

The effective arrival rates, or, throughput, $\lambda_{1}^{*}$ and $\lambda_{2}^{*}$ at each of the two stations are given as the solution to

$$
\begin{aligned}
& \lambda_{1}^{*}=\lambda_{1}+p_{2} \lambda_{2}^{*} \\
& \lambda_{2}^{*}=\lambda_{2}+p_{1} \lambda_{1}^{*}
\end{aligned}
$$

The unique solution is

$$
\begin{aligned}
& \lambda_{1}^{*}=\frac{\lambda_{1}+p_{2} \lambda_{2}}{1-p_{1} p_{2}} \\
& \lambda_{2}^{*}=\frac{\lambda_{2}+p_{1} \lambda_{1}}{1-p_{1} p_{2}}
\end{aligned}
$$

The fraction of time station 1 is empty is $P(0,1)$. Likewise, $P(1,0)$ is the fraction of time station 2 is empty. So, $(P(0,1)-P(0,0))$ is the fraction of 
time station 2 is busy while station 1 is empty. Similarly, $(P(1,0)-P(0,0))$ is the fraction of time station 1 is busy while station 2 is empty. Finally, $(1-P(1,0)-P(0,1)+P(0,0))$ is the fraction of time both stations are busy. This gives rise to the following traffic equations

$$
\begin{aligned}
\lambda_{1}^{*}= & \nu_{1}(P(1,0)-P(0,0)) \\
& +p^{*} \nu_{1}(1-P(1,0)-P(0,1)+P(0,0)) \\
\lambda_{2}^{*}= & \nu_{2}(P(0,1)-P(0,0)) \\
& +\left(1-p^{*}\right) \nu_{2}(1-P(1,0)-P(0,1)+P(0,0))
\end{aligned}
$$

or,

$$
\begin{aligned}
& \frac{\lambda_{1}^{*}}{\nu_{1}}=\left(1-p^{*}\right) P(1,0)-p^{*} P(0,1)-\left(1-p^{*}\right) P(0,0)+p^{*} \\
& \frac{\lambda_{2}^{*}}{\nu_{2}}=-\left(1-p^{*}\right) P(1,0)+p^{*} P(0,1)-p^{*} P(0,0)+1-p^{*}
\end{aligned}
$$

Adding both equations gives

$$
\frac{\lambda_{1}^{*}}{\nu_{1}}+\frac{\lambda_{2}^{*}}{\nu_{2}}=1-P(0,0)
$$

Therefore,

$$
\pi_{0,0}=1-\frac{\lambda_{1}^{*}}{\nu_{1}}-\frac{\lambda_{2}^{*}}{\nu_{2}}
$$

It is easily confirmed that (3.32) and (3.42) are equivalent forms for $\pi_{0,0}$. In summary,

\section{Lemma 3.2.1}

$$
\begin{aligned}
\pi_{0,0} & =1-\frac{\lambda_{1}\left(p_{1} \nu_{1}+\nu_{2}\right)}{\left(1-p_{1} p_{2}\right) \nu_{1} \nu_{2}}-\frac{\lambda_{2}\left(\nu_{1}+p_{2} \nu_{2}\right)}{\left(1-p_{1} p_{2}\right) \nu_{1} \nu_{2}} \\
& =1-\frac{\lambda_{1}^{*}}{\nu_{1}}-\frac{\lambda_{2}^{*}}{\nu_{2}}
\end{aligned}
$$




\subsubsection{A Relationship between $\mathbb{E} X_{1}$ and $\mathbb{E} X_{2}$}

Note that

$$
\begin{aligned}
\frac{\mathrm{d}}{\mathrm{d} y} P(\gamma(y), y)= & \gamma^{\prime}(y) \sum_{i=0}^{\infty} \sum_{j=0}^{\infty} i(\gamma(y))^{i-1} y^{j} \pi_{i, j} \\
& +\sum_{i=0}^{\infty} \sum_{j=0}^{\infty} j(\gamma(y))^{i} y^{j-1} \pi_{i, j}
\end{aligned}
$$

and, therefore,

$$
\left.\frac{\mathrm{d}}{\mathrm{d} y} P(\gamma(y), y)\right|_{y=1}=\gamma^{\prime}(1) \mathbb{E} X_{1}+\mathbb{E} X_{2}
$$

Computing $\left.\frac{\mathrm{d}}{\mathrm{d} y} P(\gamma(y), y)\right|_{y=1}$ is a laborious process that involves applying l'Hôpital's rule twice. The end result is

\section{Lemma 3.2.2}

$$
\begin{aligned}
\left(\frac{p_{1} \nu_{1}+\nu_{2}}{\nu_{1}+p_{2} \nu_{2}}\right) \mathbb{E} X_{1}+\mathbb{E} X_{2}= & \frac{p_{1} \nu_{1}+\nu_{2}}{\left(\nu_{1}+p_{2} \nu_{2}\right) \pi_{0,0}}-1 \\
& -\frac{\left[\left(1-p_{2}\right) \nu_{2}-\left(1-p_{1}\right) \nu_{1}\right]\left(p_{1} \lambda_{1}+\lambda_{2}\right)}{\left(1-p_{1} p_{2}\right) \nu_{2}\left(\nu_{1}+p_{2} \nu_{2}\right) \pi_{0,0}}
\end{aligned}
$$

Because of this relationship, only one of $\mathbb{E} X_{1}$ and $\mathbb{E} X_{2}$ needs to be calculated to have the other.

\subsubsection{Individual Forms for $\mathbb{E} X_{1}$ and $\mathbb{E} X_{2}$}

Computing the expected queue length for each queue is a key goal of the analysis of this network. In this section the preliminary principles are outlined, but fully determining $P(x, 0)$ and $P(0, y)$, along with their derivatives, as the solutions to complex boundary value problems, is required to complete the result.

Note that

$$
\frac{\mathrm{d}}{\mathrm{d} y} P(1, y)=\sum_{n=0}^{\infty} \sum_{k=0}^{\infty} k y^{k-1} \pi_{1, k}
$$


so,

$$
\mathbb{E} X_{2}=\left[\frac{\mathrm{d}}{\mathrm{d} y} P(1, y)\right]_{y=1}
$$

In the functional equation (3.13), let $x=1$, remove the common $(y-1)$ factor, take the derivative with respect to $y$ and finally let $y=1$ to give

$$
\begin{aligned}
{\left[\lambda_{2}\right.} & \left.+p^{*} p_{1} \nu_{1}-\left(1-p^{*}\right) \nu_{2}\right] \mathbb{E} X_{2}= \\
& -p_{1} \nu_{1}\left[\left(1-p^{*}\right) P(1,0)-p^{*} P(0,1)\right] \\
& +\left(1-p^{*}\right) p_{1} \nu_{1} \pi_{0,0}-\left[\lambda_{2}+p^{*} p_{1} \nu_{1}\right] \\
& +\left.p^{*}\left[p_{1} \nu_{1}+\nu_{2}\right] \frac{\mathrm{d}}{\mathrm{d} y} P(0, y)\right|_{y=1}
\end{aligned}
$$

Therefore, for $\lambda_{2} \neq\left(1-p^{*}\right) \nu_{2}-p^{*} p_{1} \nu_{1}$ (3.50) can be used to compute $\mathbb{E} X_{2}$. By the symmetry of the two-node Jackson network it is clear that

$$
\begin{aligned}
& {\left[\lambda_{1}+\left(1-p^{*}\right) p_{2} \nu_{2}-p^{*} \nu_{1}\right] \mathbb{E} X_{1}=} \\
& \quad-p_{2} \nu_{2}\left[\left(1-p^{*}\right) P(1,0)-p^{*} P(0,1)\right] \\
& \quad+p^{*} p_{2} \nu_{2} \pi_{0,0}-\left[\lambda_{1}+\left(1-p^{*}\right) p_{2} \nu_{2}\right] \\
& \quad+\left.\left(1-p^{*}\right)\left[p_{2} \nu_{2}+\nu_{1}\right] \frac{\mathrm{d}}{\mathrm{d} x} P(x, 0)\right|_{x=1}
\end{aligned}
$$

also holds. Again, $P(1,0), P(0,1)$ and $\left.\frac{\mathrm{d}}{\mathrm{d} x} P(x, 0)\right|_{x=1}$ are determined from the solution to a complex boundary value problem.

If it is not possible compute both $\mathbb{E} X_{2}$ and $\mathbb{E} X_{1}$ using (3.50) and (3.51) it suffices to calculate just one and use (3.47) to determine the other.

In the functional equation (3.13), let $y=x$, remove the common $x(x-1)$ factor and then set $x=1$. This gives,

$$
\begin{aligned}
& {\left[\nu_{1}\left(1-p_{1}\right)-\nu_{2}\left(1-p_{2}\right)\right]\left[\left(1-p^{*}\right) P(1,0)-p^{*} P(0,1)\right]=} \\
& \quad\left[\lambda_{1}+\lambda_{2}-p^{*} \nu_{1}\left(1-p_{1}\right)-\left(1-p^{*}\right) \nu_{2}\left(1-p_{2}\right)\right] \\
& \quad+\left[\left(1-p^{*}\right) \nu_{1}\left(1-p_{1}\right)+p^{*} \nu_{2}\left(1-p_{2}\right)\right] \pi_{0,0}
\end{aligned}
$$

After some tedious algebra using (3.32) for $\pi_{0,0}$ this equation reduces to

$$
\left(1-p^{*}\right) P(1,0)-p^{*} P(0,1)=\frac{p^{*}\left(\lambda_{1}+p_{2} \lambda_{2}\right)}{\left(1-p_{1} p_{2}\right) \nu_{1}}-\frac{\left(1-p^{*}\right)\left(\lambda_{2}+p_{1} \lambda_{1}\right)}{\left(1-p_{1} p_{2}\right) \nu_{2}}+1-2 p^{*}
$$


This relationship between $P(0,1)$ and $P(1,0)$ can be substituted directly into (3.50) and (3.51) to facilitate computation.

This same relationship between $P(1,0)$ and $P(0,1)$ can also be derived from the traffic equations (3.39) and (3.40). Subtracting (3.39) from (3.40) gives

$$
\left(1-p^{*}\right) P(1,0)-p^{*} P(0,1)=\frac{\lambda_{1}^{*}}{2 \nu_{1}}-\frac{\lambda_{2}^{*}}{2 \nu_{2}}+\frac{\left(1-2 p^{*}\right)}{2}\left[\pi_{0,0}+1\right]
$$

where $\lambda_{1}^{*}$ and $\lambda_{2}^{*}$ are the equivalent arrival rates at stations 1 and 2 respectively. Substituting (3.42) for $\pi_{0,0}$ gives

$$
\left(1-p^{*}\right) P(1,0)-p^{*} P(0,1)=\frac{p^{*} \lambda_{1}^{*}}{\nu_{1}}-\frac{\left(1-p^{*}\right) \lambda_{2}^{*}}{\nu_{2}}+1-2 p^{*}
$$

It is easily confirmed that (3.53) and (3.55) are equivalent.

\subsubsection{When $p^{*}=0$ or $p^{*}=1$}

When $p^{*}=0$ or $p^{*}=1$ the coupled Jackson network has some interesting operational characteristics and the computation of the mean queue lengths does not require complex analysis.

When $p^{*}=0$, the service rate of the first queue is zero whereas the second is at is full rate $\nu_{2}$. This mcans that the scrvice rate of the first queue is greater than zero only when the second queue is idle. The network will, therefore, preemptively serve the second queue over the first. With $p^{*}=0$, (3.53) simplifies to

$$
P(1,0)=1-\frac{\lambda_{2}+p_{1} \lambda_{1}}{\left(1-p_{1} p_{2}\right) \nu_{2}}=1-\frac{\lambda_{2}^{*}}{\nu_{2}}
$$

Also (3.50) reduces to

$$
\left(\lambda_{2}-\nu_{2}\right) \mathbb{E} X_{2}=-p_{1} \nu_{1} P(1,0)+p_{1} \nu_{1} \pi_{0,0}-\lambda_{2}
$$

Therefore, for $\lambda_{2} \neq \nu_{2}$,

$$
\mathbb{E} X_{2}=\frac{p_{1} \lambda_{1}^{*}+\lambda_{2}}{\nu_{2}-\lambda_{2}}
$$


Similarly, when $p^{*}=1$, the first queue is serviced preemptively over the second. Equations (3.53) and (3.51) simplify to

$$
P(0,1)=1-\frac{\lambda_{1}+p_{2} \lambda_{2}}{\left(1-p_{1} p_{2}\right) \nu_{1}}=1-\frac{\lambda_{1}^{*}}{\nu_{1}}
$$

and

$$
\left(\lambda_{1}-\nu_{1}\right) \mathbb{E} X_{1}=-p_{2} \nu_{2} P(0,1)+p_{2} \nu_{2} \pi_{0,0}-\lambda_{1}
$$

respectively. Therefore, if $\lambda_{1} \neq \nu_{1}, \mathbb{E} X_{1}$ can be directly calculated as

$$
\mathbb{E} X_{1}=\frac{p_{2} \lambda_{2}^{*}+\lambda_{1}}{\nu_{1}-\lambda_{1}}
$$

\subsection{The Boundary Value Problem}

The complete analysis of this coupled Jackson network requires the full specification of $P(x, y)$. From the functional equation (1.3) it is clear that $P(x, y)$ can be represented as a combination of $P(0, y)$ and $P(x, 0)$. In the following analysis it is shown how $P(0, y)$ can be determined as the solution to a complex boundary value problem. While not specifically presented, $P(x, 0)$ can be determined in an identical manner.

In the following, a subset from the zero-space of the kernel $h_{1}(x, y)$ is selected such that it forms a closed contour in the complex space. It is shown that $P(0, y)$ is analytic and its value can be fully defined along this contour. A boundary value problem is then formulated the solution to which gives $P(0, y)$ within the region bounded by the contour.

\subsubsection{Analysis of the Kernel}

The kernel $h_{1}(x, y)$ to the functional equation can be viewed as a polynomial of degree 2 in $y$. For every value of $x$, therefore, there are two possible values, say $y_{+}(x)$ and $y_{-}(x)$, such that $h_{1}\left(x, y_{+}(x)\right)=h_{1}\left(x, y_{-}(x)\right)=0$.

It is possible to rewrite $h_{1}(x, y)$ as

$$
\begin{aligned}
h_{1}(x, y)= & -\left(\lambda_{2} x+p^{*} p_{1} \nu_{1}\right) y^{2} \\
& -\left[p^{*}\left(1-p_{1}\right) \nu_{1}-\left(\lambda_{1}+\lambda_{2}+p^{*} \nu_{1}+\left(1-p^{*}\right) \nu_{2}\right) x+\lambda_{1} x^{2}\right] y \\
& -\left[\left(1-p^{*}\right) p_{2} \nu_{2} x^{2}+\left(1-p^{*}\right)\left(1-p_{2}\right) \nu_{2} x\right]
\end{aligned}
$$


The zeros of the kernel are given by the two valued function

$$
y(x)=-\frac{s_{1}(x) \pm \sqrt{D_{1}(x)}}{2\left(\lambda_{2} x+p^{*} p_{1} \nu_{1}\right)}
$$

where et us define

$$
\begin{aligned}
r & =\lambda_{1}+\lambda_{2}+p^{*} \nu_{1}+\left(1-p^{*}\right) \nu_{2} \\
s_{1}(x) & =p^{*}\left(1-p_{1}\right) \nu_{1}-r x+\lambda_{1} x^{2} \\
D_{1}(x) & =\left[s_{1}(x)\right]^{2}-4\left(1-p^{*}\right) \nu_{2} x\left(p_{2} x+1-p_{2}\right)\left(\lambda_{2} x+p^{*} p_{1} \nu_{1}\right)
\end{aligned}
$$

The zero-space of the kernel, within the unit circle, is therefore given by $\{(x, y):|x| \leq 1, y=y(x)\}$.

Note that the following analysis assumes that $0<p^{*}<1$ as the boundary value technique cannot be used if $p^{*}=0,1$. If $p^{*}=0$ then $h_{3}(x, y)=0$ and from the functional equation (1.3) there fails to be a relationship between $P(x, y)$ and $P(0, y)$. If $p^{*}=1$ then $y(x)$ is never complex for real $x$.

Lemma 3.3.1 The algebraic function $y(x)$, defined by $h_{1}(x, y(x))=0$ has two real branch points $0 \leq x_{1}<x_{2} \leq 1$ for $x \in[0,1]$.

Proof: The branch points (i.e. the points where the derivative is undefined) are the zeros of the discriminant $D_{1}(x)$. There are two zeros of $D_{1}(x)$ for $x \in[0,1]$.

Let $x^{\prime}$ be the smallest zero of $s_{1}(x)$; i.e.

$$
x^{\prime}= \begin{cases}\frac{r-\sqrt{r^{2}-4 p^{*}\left(1-p_{1}\right) \lambda_{1} \nu_{1}}}{2 \lambda_{1}} & \lambda_{1}>0 \\ \frac{p^{*}\left(1-p_{1}\right) \nu_{1}}{r} & \lambda_{1}=0\end{cases}
$$

Note that

$$
\begin{aligned}
r^{2}-4 p^{*}\left(1-p_{1}\right) \lambda_{1} \nu_{1} & =\left(\lambda_{1}+\lambda_{2}+p^{*} \nu-1+\left(1-p^{*}\right) \nu_{2}\right)^{2}-4 p^{*}\left(1-p_{1}\right) \lambda_{1} \nu_{1} \\
& =\left(\lambda_{1}+\lambda_{2}-p^{*} \nu-1+\left(1-p^{*}\right) \nu_{2}\right)^{2}+4 p^{*} p_{1} \lambda_{1} \nu_{1} \\
& >0
\end{aligned}
$$

so, $x^{\prime}$ is indeed real.

First consider $p_{1} \neq 1$. It is seen that $s_{1}(x) \geq 0$ for $x \leq 0$, and $s_{1}(1)<0$. Therefore, it must be true that $0<x^{\prime}<1$. 

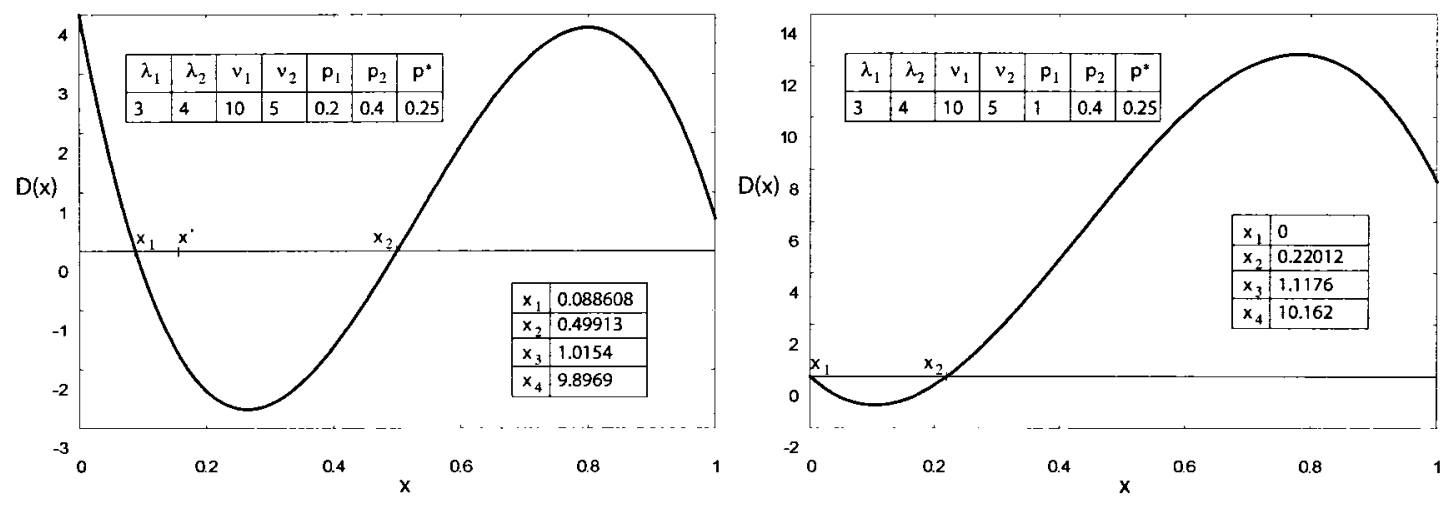

Figure 3.3: Coupled Jackson: Discriminant as a Function of Real $x$

Now, $D_{1}\left(x^{\prime}\right)=-4\left(\lambda_{2} x+p^{*} p_{1} \nu_{1}\right)\left(1-p^{*}\right) \nu_{2}\left[p_{2} x+1-p_{2}\right] x<0$ for $x>0$. Also, $D_{1}(0)=s_{1}(0)>0$, and

$$
D_{1}(1)=\left(\lambda_{2}+p^{*} p_{1} \nu_{1}-\left(1-p^{*}\right) \nu_{2}\right)^{2} \geq 0
$$

It must concluded that $D_{1}(x)$ has two real zeros for $x \in[0,1]$ with $0<x_{1}<$ $x^{\prime}<x_{2} \leq 1$.

Now, if $p_{1}=1$ then $x^{\prime}=0$; so, $x_{1}=x^{\prime}=0$. The fact that $D_{1}^{\prime}(0)<0$ and $D_{1}(1) \geq 0$ requires a second root; i.e. $0=x_{1}<x_{2} \leq 1$.

Corollary 3.3.1 If $p_{1}=1$ then $x_{1}=0$, otherwise, $0<x_{1}<x_{2}$.

Proof: $D_{1}(0)=0$ if and only if $p_{1}=1$.

Figure 3.3 graphs the discriminant of $y(x)$ for two parameter sets. Note how $x_{1}=0$ when $p_{1}=1$.

The following two lemmas are not required for any of the results presented in this thesis. However, they are key to describing the asymptotic behavior of the network. They are therefore included to completely describe the branch points of $h_{1}(x, y)$ for the sake of future research.

Lemma 3.3.2 If $\lambda_{1}>0$, the algebraic function $y(x)$, defined by $h_{1}(x, y(x))=$ 0 , has two real branch points $1 \leq x_{3}<x_{4}$ for $x \geq 1$. 
Proof: With $\lambda_{1}>0, D_{1}(x)$ is a polynomial of degree four. Two roots have already been found: $x_{1}$ and $x_{2}$, so, two more remain: $x_{3}$ and $x_{4}$.

Let $x^{\prime \prime}$ be the largest of the two zeros of $s_{1}(x)$. $x^{\prime \prime}$ is expressed as

$$
x^{\prime \prime}=\frac{r+\sqrt{r^{2}-4 p^{*}\left(1-p_{1}\right) \lambda_{1} \nu_{1}}}{2 \lambda_{1}}
$$

Clearly, $s_{1}(1)<0$, and as $x \rightarrow+\infty, s_{1}(x) \rightarrow+\infty$. Therefore, $x^{\prime \prime}>1$.

From, $D_{1}\left(x^{\prime \prime}\right)<0, D_{1}(x) \rightarrow+\infty$ as $x \rightarrow+\infty$ and $D_{1}(1) \geq 0$, it can be concluded that $D_{1}(x)$ has two real roots with $1 \leq x_{3} \leq x^{\prime \prime}<x_{4}$ for $x \geq 1$.

Lemma 3.3.3 If $\lambda_{1}=0$ then $y(x)$ has one real branch point $1 \leq x_{3}$ for $x \geq 1$

Proof: With $\lambda_{1}=0, D_{1}(x)$ is a polynomial of degree three. Already $x_{1}$ and $x_{2}$ have been found, so, $x_{3}$ must remain.

As $D_{1}(1) \geq 0$ and $D_{1}(x) \rightarrow-\infty$ as $x \rightarrow+\infty$, so, $1 \leq x_{3}$.

\section{Corollary 3.3.2 If}

$\lambda_{2}=\left(1-p^{*}\right) \nu_{2}-p^{*} p_{1} \nu_{1}$ and $\begin{cases}\lambda_{1}=p^{*} \nu_{1}-\left(1-p^{*}\right) p_{2} \nu_{2} & \text { then } x_{2}=1=x_{3} \\ \lambda_{1}<p^{*} \nu_{1}-\left(1-p^{*}\right) p_{2} \nu_{2} & \text { then } x_{2}=1<x_{3} \\ \lambda_{1}>p^{*} \nu_{1}-\left(1-p^{*}\right) p_{2} \nu_{2} & \text { then } x_{2}<1=x_{3}\end{cases}$

Proof: From (3.68) it is clear that $D_{1}(1)=0$ when $\lambda_{2}=\left(1-p^{*}\right) \nu_{2}-p^{*} p_{1} \nu_{1}$. This condition requires either $x_{2}=1$ or $x_{3}=1$.

With $\lambda_{2}=\left(1-p^{*}\right) \nu_{2}-p^{*} p_{1} \nu_{1}$, the derivative of $D_{1}(x)$ with respect to $x$, at $x=1$, is

$$
D_{1}^{\prime}(1)=-4\left(1-p^{*}\right) \nu_{2}\left[\lambda_{1}+\left(1-p^{*}\right) p_{2} \nu_{2}-p^{*} \nu_{1}\right]
$$

So, if $\lambda_{1}=p^{*} \nu_{1}-\left(1-p^{*}\right) p_{2} \nu_{2}$ then $D_{1}^{\prime}(1)=0$ and $x=1$ must be a local maximum of $D_{1}(x)$. In this case, $x=1$ must be a second order root of $D_{1}(x)$; i.e. $x_{2}=x_{3}=1$.

If $\lambda_{1}<p^{*} \nu_{1}-\left(1-p^{*}\right) p_{2} \nu_{2}$ then $D_{1}^{\prime}(1)>0$. If $\lambda_{1}>0$ we know that $D_{1}\left(x^{\prime \prime}\right)<0$ with $x_{3}<x^{\prime \prime}<x_{4}$; so, $x_{3}>1$. If $\lambda_{1}=0$ then $D_{1}(x) \rightarrow-\infty$ as $x \rightarrow+\infty$; 

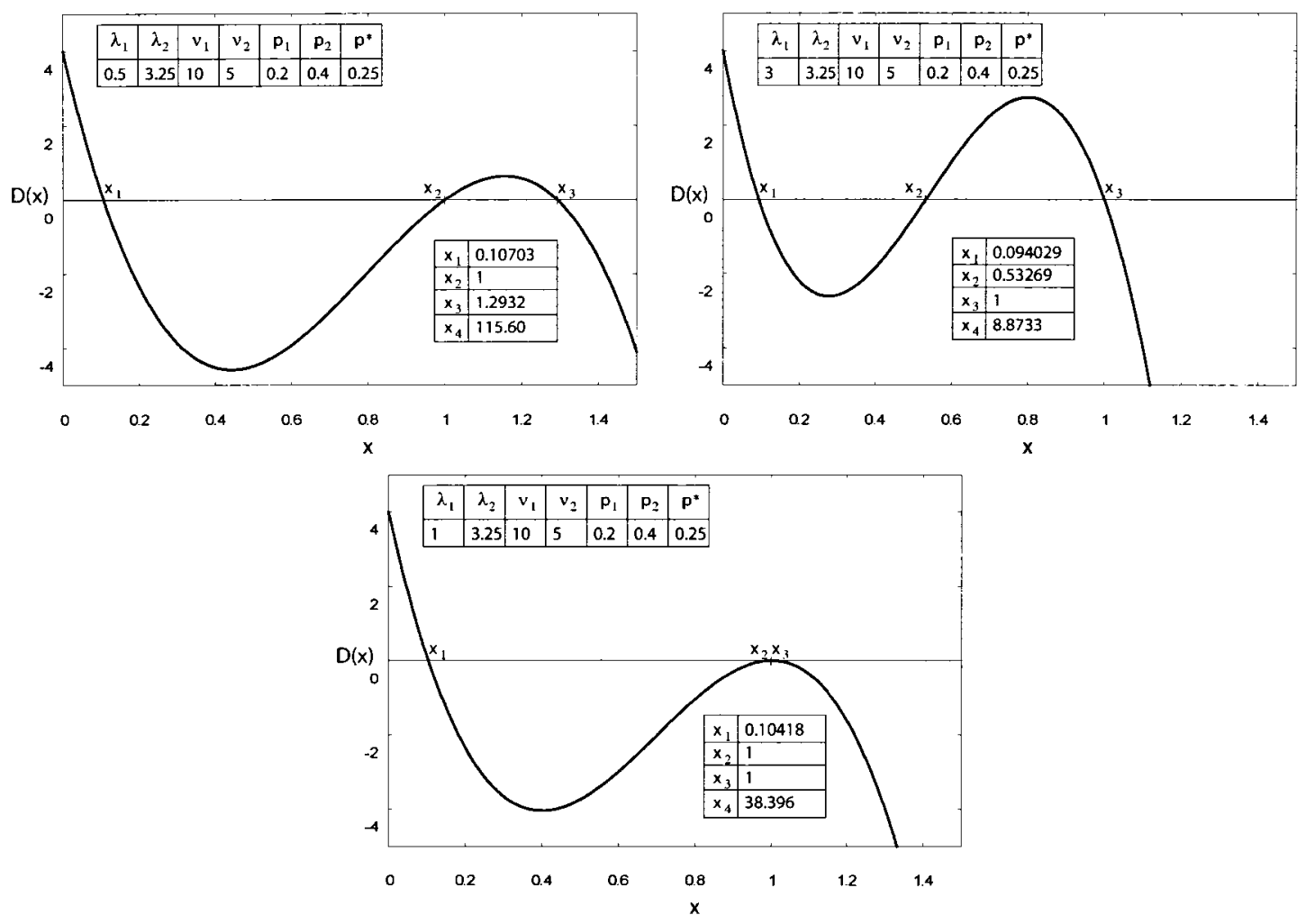

Figure 3.4: Coupled Jackson: Three options for $D(1)=0$

therefore, $x_{3}>1$.

Likewise, if $\lambda_{1}>p^{*} \nu_{1}-\left(1-p^{*}\right) p_{2} \nu_{2}$ then $D_{1}^{\prime}(1)<0$. In order to have $D_{1}\left(x^{\prime}\right)<0$ with $x^{\prime}<x_{2}$ it requires $x_{2}<1$.

The result of this corollary is nicely illustrated in Figure 3.4. In all three graphs, $\lambda_{2}=\left(1-p^{*}\right) \nu_{2}-p^{*} p_{1} \nu_{1}$ so the discriminant always has a zero at $x=1$. However, by varying $\lambda_{1}$ the zero at $x=1$ varies between being the second and third branch point of $y(x)$.

Now that the branch points of the kernel have been fully described, a subset of the complete zero-space is selected. Namely, $x$ is chosen to be strictly real and lie in the interval $\left[x_{1}, x_{2}\right]$. The function $y(x)$ then defines a closed contour in the complex space. 
Lemma 3.3.4 For each $x \in\left[x_{1}, x_{2}\right], y(x)$ lies on the closed contour $L$, which is symmetric with respect to the real line, and defined by

$$
|y|^{2}=m(\operatorname{Re}(y))
$$

where

$$
m(\delta)=\frac{\left(1-p^{*}\right) \nu_{2} \zeta(\delta)\left[p_{2} \zeta(\delta)+1-p_{2}\right]}{\lambda_{2} \zeta(\delta)+p^{*} p_{1} \nu_{1}}
$$

and

$$
\zeta(\delta)= \begin{cases}\frac{r-2 \lambda_{2} \delta-\sqrt{\left[r-2 \lambda_{2} \delta\right]^{2}-4 p^{*} \lambda_{1} \nu_{1}\left[1-p_{1}+2 p_{1} \delta\right]}}{2 \lambda_{1}} & \lambda_{1}>0 \\ \frac{p^{*}\left(1-p_{1}+2 \delta p_{1}\right) \nu_{1}}{r-2 \delta \lambda_{2}} & \lambda_{1}=0\end{cases}
$$

It further holds that

$$
|y|^{2} \leq \alpha
$$

where

$$
\alpha=\sqrt{\frac{\left(1-p^{*}\right) \nu_{2} x_{2}\left(p_{2} x_{2}+1-p_{2}\right)}{\lambda_{2} x_{2}+p^{*} p_{1} \nu_{1}}}
$$

Proof: For $x \in\left(x_{1}, x_{2}\right), D_{1}(x)$ is negative, so $y_{1}(x)$ and $y_{2}(x)$ are complex conjugates. It also follows that

$$
\operatorname{Re}(y(x))=-\frac{s_{1}(x)}{2\left(\lambda_{2} x+p^{*} p_{1} \nu_{1}\right)}
$$

and

$$
\operatorname{Im}(y(x))= \pm \frac{\sqrt{4\left(1-p^{*}\right) \nu_{2} x\left(p_{2} x+1-p_{2}\right)\left(\lambda_{2} x+p^{*} p_{1} \nu_{1}\right)-\left[s_{1}(x)\right]^{2}}}{2\left(\lambda_{2} x+p^{*} p_{1} \nu_{1}\right)}
$$

so,

$$
|y(x)|^{2}=\frac{\left(1-p^{*}\right) \nu_{2} x\left(p_{2} x+1-p_{2}\right)}{\lambda_{2} x+p^{*} p_{1} \nu_{1}}
$$



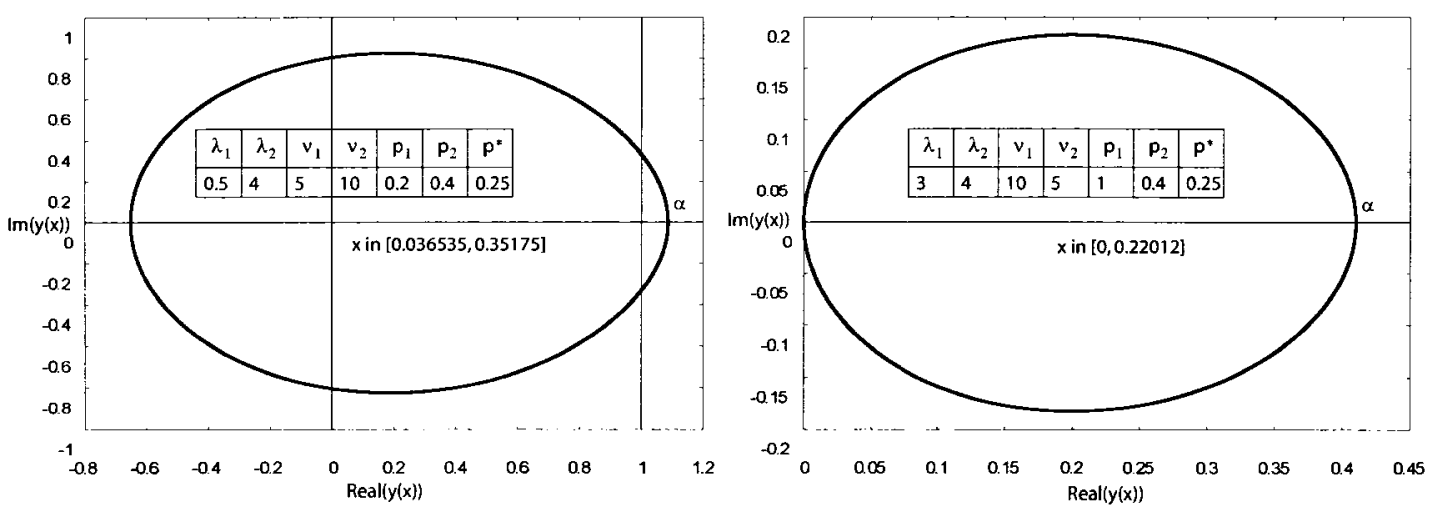

Figure 3.5: Coupled Jackson: The Contour $L$

$\zeta(\delta)$ is derived by solving (3.74) for $x$ with $\delta=\operatorname{Re}(y(x))$ and taking the solution such that $x \in[0,1]$. Note that $x^{\prime}=\zeta(0)$, where $s_{1}\left(x^{\prime}\right)=0$.

As, $\frac{\mathrm{d}}{\mathrm{d} x}|y(x)|>0$ for $x>0,|y(x)|$ is increasing for $x>0$; so, $\max |y(x)|=$ $y\left(x_{2}\right)=\alpha$.

Figure 3.5 illustrates the contour $L$ for two sets of parameters. Note how in one case the contour is entirely contained within the unit circle but in the other case it is not. The following two corollaries explain this behavior.

Corollary 3.3.3 Let $L^{+}$be the interior of the closed contour L. It is always true that $0 \in L \cup L^{+}$.

Let us define

$\xi(y)= \begin{cases}\frac{\lambda_{2}|y|^{2}-\left(1-p^{*}\right)\left(1-p_{2}\right) \nu_{2}+\sqrt{\left[\lambda_{2}|y|^{2}-\left(1-p^{*}\right)\left(1-p_{2}\right) \nu_{2}\right]^{2}+4 p^{*}\left(1-p^{*}\right) p_{1} p_{2} \nu_{1} \nu_{2}|y|^{2}}}{2\left(1-p^{*}\right) p_{2} \nu_{2}} & 0<p_{2} \leq 1 \\ \frac{p^{*} p_{1} \nu_{1}|y|^{2}}{\left(1-p^{*}\right) \nu_{2}-\lambda_{2}|y|^{2}} & p_{2}=0\end{cases}$

If $x_{2}=\xi(1)$ then $1 \in L$. For greater values of $x_{2}, 1 \in L^{+}$. For lesser values of $x_{2}, 1 \notin L \cup L^{+}$.

Proof: For all $x \in\left[x_{1}, x_{2}\right], y(x) \in L$. As, $L$ is a closed contour, symmetric with the real axis, $\operatorname{Re}(y(x)) \in L \cup L^{+}$. In the previous lemma $x^{\prime} \in\left[x_{1}, x_{2}\right]$ 
was found such that $s_{1}\left(x^{\prime}\right)=0$ and therefore $\operatorname{Re}\left(y\left(x^{\prime}\right)\right)=0$. It clearly follows that $0 \in L \cup L^{+}$.

Solving (3.76) for $x$ and taking the solution in $[0,1]$ gives $\xi(y)$. From the previous lemma it is clear that $x_{2}=\xi(\alpha)$. Logically, $\alpha=1$ only if $x_{2}=\xi(1)$. Lesser values of $x_{2}$ result in $\alpha<1$ and greater values result in $\alpha>1$. Clearly, if $\alpha=1$ then $1 \in L$, if $\alpha>1$ then $1 \in L^{+}$, and if $\alpha<1$ then $1 \notin L \cup L^{+}$.

\section{Corollary 3.3.4}

$$
\begin{aligned}
\lambda_{2}=\left(1-p^{*}\right) \nu_{2}-p^{*} p_{1} \nu_{1} & \Rightarrow \alpha=1 \\
\lambda_{2}>\left(1-p^{*}\right) \nu_{2}-p^{*} p_{1} \nu_{1} & \Rightarrow \alpha<1 \\
\alpha>1 & \Rightarrow \lambda_{2}<\left(1-p^{*}\right) \nu_{2}-p^{*} p_{1} \nu_{1}
\end{aligned}
$$

Proof: From (3.76) it is revealed that $|y(x)|$ is increasing in $x>0$. Therefore, $\alpha=y\left|x_{2}\right|$ where $x_{2} \leq 1$. When $\lambda_{2}=\left(1-p^{*}\right) \nu_{2}-p^{*} p_{1} \nu_{1}, x_{2}=1$ and, $\alpha=1$. In general,

$$
\alpha \leq \sqrt{\frac{\left(1-p^{*}\right) \nu_{2}}{\lambda_{2}+p^{*} p_{1} \nu_{1}}}
$$

So, when $\lambda_{2}>\left(1-p^{*}\right) \nu_{2}-p^{*} p_{1} \nu_{1}$ it must be true that $\alpha<1$. Finally, the remaining range of values for $\lambda_{2}$, namely, $\lambda_{2}<\left(1-p^{*}\right) \nu_{2}-p^{*} p_{1} \nu_{1}$ is a necessary, but not sufficient, condition for $\alpha>1$.

\subsubsection{Analytic Continuation}

To conclude that $P(0, y)$ is analytic within the region bounded by the contour $L$ even if the contour escapes the unit circle, it is necessary to analytically continue $P(0, y)$ from the unit circle to the larger space $L^{+}$.

Lemma 3.3.5 The function $P(0, y)$ is analytic in the domain $L^{+}$and satisfies for $y \in L$ the condition

$$
\operatorname{Im}[P(0, y)]=-\operatorname{Im}\left[-P(0,0) \frac{h_{4}(\xi(y), y)}{h_{3}(\xi(y), y)}\right]
$$

if $h_{3}(x, y) \neq 0$ for $y \in L^{+}$and $|x|<1$ when $\alpha>1$. 
Proof: For zero-pairs $(x, y)$ of the kernel $h_{1}(x, y)$ for which $P(x, y)$ is finite,

$$
h_{2}(x, y) P(x, 0)+h_{3}(x, y) P(0, y)+h_{4}(x, y) P(0,0)=0
$$

or,

$$
P(0, y)=\frac{1-p^{*}}{p^{*}} P(x, 0)-\frac{h_{4}(x, y)}{h_{3}(x, y)} P(0,0)
$$

For $(x, y)$ such that $y \in L, x=\xi(y)$. Therefore, (3.79) follows from the fact that $P(x, 0)$ is finite and real for $x \in\left[x_{1}, x_{2}\right]$.

By definition, $P(0, y)$ is analytic within the unit circle. If $\alpha \leq 1$ then $L$ lies entirely within the unit circle and, therefore, $P(0, y)$ is clearly analytic in $L^{+}$. If $\alpha>1$, as in the proof of Lemma 2.3.3, it is required to show that the right hand side of $(3.80)$ is analytic when $y \in L^{+}$. This is ensured by the closing condition of the Lemma (this condition can probably be removed with further research).

The conditions necessary to establish a boundary value problem are now in place.

\subsubsection{The Boundary Value Problem}

The theory behind the following statements is fully developed in Cohen \& Boxma's text [2] section I.3.

The determination of $P(0, y)$ reduces to the solution of the following RiemannHilbert boundary value problem on the contour $L$ : Determine a function $P(0, y)$ such that

1. $P(0, y)$ is analytic for $y \in L^{+}$and continuous for $y \in L \cup L^{+}$.

2. $\operatorname{Re}[i P(0, y)]=c(y)$, for $y \in L$

where

$$
c(y)=\operatorname{Im}\left[P(0,0) \frac{h_{4}(\xi(y), y)}{h_{3}(\xi(y), y)}\right]
$$

The standard way to solve this type of boundary value problem is to transform the boundary condition of (3.79) to a condition on the unit circle. The conformal mapping

$$
z=f(y): L^{+} \rightarrow C^{+}
$$


and its inverse

$$
y=f_{0}(z): C^{+} \rightarrow L^{+}
$$

is introduced, where $C^{+}$is the interior of the unit circle $C$. Using these mappings the Riemann-Hilbert problem on $L$ reduces to a Dirichlet problem on the unit circle. The solution to this problem gives

$$
P(0, y)=-\frac{1}{2 \pi} \oint_{C} c\left(f_{0}(w)\right) \frac{w+f(y)}{w-f(y)} \frac{\mathrm{d} w}{w}+\kappa_{1}, \quad y \in L \cup L^{+}
$$

where $\kappa_{1}$ is a constant. As $\pi_{0,0}$ is known from (3.32), $\kappa_{1}$ can easily be calculated by evaluating (3.82) for $y=0$.

For $y \in L \cup L^{+}$the derivative of $P(0, y)$ can also be found as

$$
\frac{\mathrm{d}}{\mathrm{d} y}=-\frac{1}{\pi} \oint_{C} c\left(f_{0}(\omega)\right) \frac{f^{\prime}(\omega)}{(\omega-f(y))^{2}} \mathrm{~d} \omega
$$

The technique outlined in section 2.3.4 can be applied in an identical fashion to compute the conformal map $f$, its inverse $f_{0}$, and its derivative $f^{\prime}$.

\subsection{Conclusion}

As the solution to a boundary value problem, therefore, $P(0, y)$ has be derived. By the symmetry of the Jackson network, $P(x, 0)$ can be found in an identical manner. In the preceding analysis for $P(0, y)$ if the following are swapped

$$
\begin{aligned}
x & \Leftrightarrow y \\
\lambda_{1} & \Leftrightarrow \lambda_{2} \\
\nu_{1} & \Leftrightarrow \nu_{2} \\
p^{*} & \Leftrightarrow 1-p^{*}
\end{aligned}
$$

the analysis applies equally well to $P(x, 0)$.

Having both $P(x, 0)$ and $P(0, y)$ means that $P(x, y)$ is fully specified and the analysis is complete. Additionally, from the derivatives of $P(x, 0)$ and $P(0, y)$, the expected queue lengths can be calculated.

Further research on this model could be rewarding. 
1. Tail asymptotics can perhaps be derived by considering the behavior of the characteristic function between the second and third branch points of the kernel.

2. A means to evaluate $P(x, 0)$ and $P(0, y)$ (and also their derivatives) at $x=1$ and $y=1$, respectively, when the contour used in solving the boundary problem is contained within the unit circle, needs to be developed

The complexity of this model, however, makes any sort of analysis prohibitively difficult. 


\section{Bibliography}

[1] Blanc, J. P. C.: The Relaxation Time of Two Queueing Systems in Series. Communications in Statistics - Stochastic Models 1 (1985), 116.

[2] Cohen, J. W., Boxma, O. J.: Boundary Value Problems In Queueing System Analysis. Amsterdam: North Holland (1983).

[3] Fayolle, G.: Methodes Analytiques pour les Files d'Attente Couplées. Ph.D. thesis, Université de Paris VI (1979).

[4] Fayolle, G., Iasnogorodski, R.: Two coupled processors: The reduction to a Riemann-Hilbert problem. Zeitschrift für Wahrscheinlichkeitstheorie und Verwandte Gebiete 47 (1979) 325-351.

[5] Flatto, L., McKean, H., P.: Two Queues in Parallel. Communications on Pure and Applied Mathematics 30 (1977), 255-263.

[6] Foley, R. D., McDonald, D. R.: Large Deviations of a Modified Jackson Network: Stability and Rough Asymptotics. The Annals of Applied Probability 15 (2005) 519-541.

[7] Haight, F., A.: Two Queues in Parallel. Biometrika 45 (1958) 401-410.

[8] Iasnogorodski, R.: Problèmes-Frontières dans les Files d'Attente. Ph.D. thesis, Université de Paris VI (1979).

[9] Kingman, J., F., C.: Two Similar Queues in Parallel. Annals of Mathematics and Statistics 32 (1961) 1314-1323.

[10] Kober, H.: Dictionary of Conformal Representations. Dover, New York (1957). 
[11] van Leeuwaarden, J. S. H.: Queueing Models for Cable Access Networks. Ph.D. thesis, Technische Universiteit Eindhoven (2005).

[12] van Leeuwaarden, J. S. H., Resing, J. A. C.: A Tandem Queue with Coupled Processors: Computational Issues. Queueing Systems: Theory and Applications 51 (October 2005) 29-52.

[13] Resing, J., Örmeci, L.: A tandem queueing model with coupled processors. Operations Research Letters 31 (2003), 383-389. 